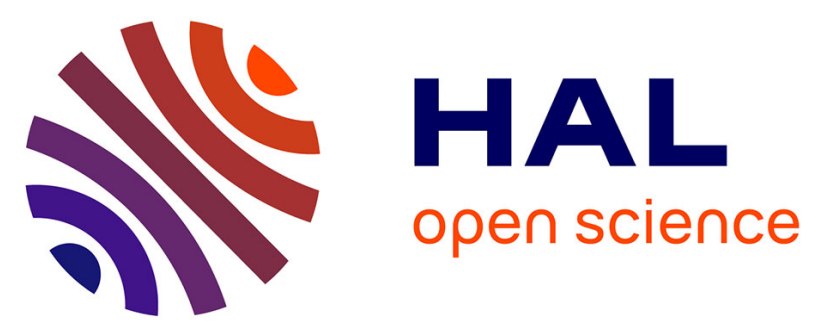

\title{
A Discrete-Time PID-like Consensus Control: Application to the Wind Farm Distributed Control Problem
}

Nicolo Gionfra, Guillaume Sandou, Houria Siguerdidjane, Damien Faille, Philippe Loevenbruck

\section{To cite this version:}

Nicolo Gionfra, Guillaume Sandou, Houria Siguerdidjane, Damien Faille, Philippe Loevenbruck. A Discrete-Time PID-like Consensus Control: Application to the Wind Farm Distributed Control Problem. O. Gusikhin and K. Madani. Informatics in Control, Automation and Robotics, 495, pp.106-134, 2019, Lecture Notes in Electrical Engineering, 10.1007/978-3-030-11292-9_6 . hal-02861064

\section{HAL Id: hal-02861064 \\ https://hal-centralesupelec.archives-ouvertes.fr/hal-02861064}

Submitted on 8 Jun 2020

HAL is a multi-disciplinary open access archive for the deposit and dissemination of scientific research documents, whether they are published or not. The documents may come from teaching and research institutions in France or abroad, or from public or private research centers.
L'archive ouverte pluridisciplinaire HAL, est destinée au dépôt et à la diffusion de documents scientifiques de niveau recherche, publiés ou non, émanant des établissements d'enseignement et de recherche français ou étrangers, des laboratoires publics ou privés. 


\title{
A Discrete-Time PID-like Consensus Control: Application to the Wind Farm Distributed Control Problem
}

\author{
Nicolò Gionfra ${ }^{1}$, Guillaume Sandou, Houria Siguerdidjane, \\ Damien Faille ${ }^{2}$, and Philippe Loevenbruck ${ }^{3}$ \\ 1 Laboratoire des Signaux et Systèmes (L2S, CentraleSupélec, Université \\ Paris-Saclay), 91192 Gif-sur-Yvette, France, \\ \{nicolo.gionfra, guillaume.sandou, \\ houria.siguerdidjane\}@centralesupelec.fr \\ 2 EDF R\&D, Department PRISME, 78401 Chatou, France, \\ damien.faille@edf.fr \\ 3 EDF R\&D, Department EFESE, 91120 Palaiseau, France, \\ philippe.loevenbruck@edf.fr
}

\begin{abstract}
The problem of discrete-time multi-agent systems governed by general MIMO dynamics is addressed. By employing a PID-like distributed protocol, we aim to solve two relevant consensus problems, namely the leaderless weighted consensus under disturbances and leaderfollower weighted consensus under time-varying reference state. Sufficient conditions for stability as well as a LMI approach to tune the controller gains are provided. The two consensus techniques are then applied to solve two issues concerning the wind farm (WF) power maximization problem under wake effect. Leaderless consensus aims at averaging out zero-mean wind disturbance effects on the optimal WF power sharing, while leader-follower control is employed to restore it in the case of power reference errors. Simulations are carried out on a small WF example, whose units are the NREL's CART wind turbines.
\end{abstract}

Keywords: consensus control, multi-agent systems, wind farm, wake effect

\section{Introduction}

In recent years much research effort has been devoted to the area of multiagent cooperative control because of its wide range of applications and potential benefits. Cooperation of a coordinated multi-agent network is sought via distributed algorithms as they present some interesting advantages over their centralized counterpart, e.g. avoiding single point of failure, reducing communication and computational burden, etc. The main problem in distributed coordination, known as consensus problem, is the one of achieving an agreement on some variables of interest, named coordination variables, of each agent via 
local interactions. These variables evolve according to a prescribed dynamics describing the physics of the problem, while interactions among agents are defined by a given communication graph. Finding a distributed protocol to solve the aforementioned problem has been extensively treated for single and double integrator dynamic agents, e.g. [1]. However, in a more general framework, general dynamics need to be considered in order to describe the agents behavior.

The consensus problem for this latter case has been discussed for both continuous and discrete-time multi-agent systems. In addition, it can be further divided in two main classes of problems, namely leaderless and leader-follower ones. As far as the former is concerned, the most employed distributed protocol is given by a static state feedback law, also called P-like distributed control. One can cite, for instance, $[2-4]$ for the continuous-time framework, and $[3,5-7]$ for the discrete one, where the consensus problem is led back to the one of simultaneously stabilizing multiple LTI systems. References $[3,6]$ also solve a leaderfollower problem where the leader has an autonomous time-invariant dynamics. Another interesting problem is the one of finding the optimal P-like protocol gain in order to improve consensus under system uncertainties, as in [8], and disturbances as in $[9,10]$, for continuous time systems, and [11] for discrete-time ones. The proposed approaches usually make use of some $\mathcal{H}_{2}$ or $\mathcal{H}_{\infty}$ constraints to be respected, and they are in general more involved than the one of simultaneously stabilizing multiple systems. For instance, [10] provide necessary and sufficient conditions, for the continuous-time case to solve the consensus problem while guaranteeing some properties on the aforementioned norms. On the other hand, for discrete-time systems only sufficient conditions are provided using results from robust control as in [11]. Dynamic distributed controllers are also proposed for consensus achievement based on local output measurements, e.g. [3]. In the continuous-time framework, [12] provide a controller with limited energy, while a general full order one is presented in [13] to achieve some $\mathcal{H}_{\infty}$ performance. Other possible structures have been explored too. Indeed, given the common P-like controller, one can easily think of a more general PID-like structure. In continuous-time, for instance, [14] propose a PI-like distributed algorithm for single integrator dynamic agents, and [15] provide a PID-like controller for general high-order SISO systems. Similar control design is applied to solve a leader-follower consensus under time-varying reference state, as in [16], and in its sampled-data counterpart [17], where a PD-like protocol is given. Even though the presented literature review is nowhere near exhaustive, one can remark that poorer attention has been devoted to discrete-time dynamic protocols for general LTI MIMO systems, and this is on what we wish to focus our attention in the sequel.

In this chapter our first contribution concerns the proposal of a PID-like distributed controller for the aforementioned systems, and we provide a possible way of tuning the controller parameters based on the solution of LMIs. The results here presented have been object of our previous work in [18], where we treated the problems of leaderless consensus under the presence of disturbances, and leader-follower consensus under a time-varying reference state. Differently from 
the aforesaid work, here we propose a generalized control technique in order to treat weighted consensus problems, i.e. those multi-agent systems in which consensus has to respect predefined gains, which determine given distances among the coordination variables. Despite being similar to what we already presented, this additional step reveals to be necessary to tackle a great variety of real-world problems such as the one considered in this chapter, namely the wind farm distributed control problem.

Our second main scope of this work is indeed concerned with applying the proposed consensus techniques for the sake of controlling a wind farm. In particular, we focus on the issues related to the power maximization problem for those wind farms experiencing the so-called wake effect. In such case, it turns out that considering the aerodynamic coupling among the wind turbines (WTs) leads to potential power gains when maximizing the power production, (see e.g. [19]), and justifies a growing interest in cooperative methods to control them. Typically the problem of power maximization under wake interaction is handled via a first step of optimization under the assumption of a static system. This approximation is mainly due to the high nonconvexity of the wake model that makes the problem hard to be treated directly under a control perspective. Here, cooperation is reached by considering a common WF optimization problem among the WTs rather than a more classic greedy WT optimization such as the maximum power tracking point (MPPT) operating mode. The overall WF control architecture thus exhibits a two-layer hierarchical structure, where the higher control level is concerned with providing optimal power references to the local WT controllers. These are then operated in decentralized mode, i.e. once received the power reference to track, no additional cooperation is performed among the WT controllers. In this chapter we claim that allowing additional cooperation also at the WT control level can lead to interesting benefits. In particular we show how cooperation at the lower level can be achieved via the consensus control techniques object of the first part of this chapter. Thus, the lower control considered in this work is thus said to be distributed.

It is important to point out that wind farm power maximization can be alternatively seen as the problem of finding the optimal power sharing of the available wind source among the WTs. Similar power sharing problems for wind farms have been treated in [20-22]. Based on the common assumption that the available wind power is higher than the demanded one, and with no wake effect consideration, they employ different distributed control approaches to deal with the problem of meeting the desired WF power output. The distributed WF control approach here presented addresses the problem of allowing proper power sharing among the WTs, enhancing the respect of the imposed higher level power gains despite the system dynamics and the presence of wind disturbances. Even if it has some common ideas, it substantially differs from the mentioned references either in the problem addressed and in the control techniques proposed to tackle it. The developments here proposed have been suggested in our preliminary work of [23]. Nonetheless, in this chapter, these are extended by considering new wind farm control applications and the theorems shown therein are here provided with 
the according proof.

The remainder of the chapter is organized as follows. Sections 2 and 3 are devoted to the leaderless and leader-follower techniques respectively. The wind farm control problem is introduced in Section 4, while its control design is described in Section 5. Simulations are shown in Section 6. The chapter ends with conclusions and future perspectives in Section 7 .

\section{Leaderless Consensus Under the Presence of Disturbances}

\subsection{Problem Formulation}

In the sequel, the reader may refer to Appendix 1 for basic notions and definitions concerning graph theory. We consider $N$ identical agents governed by general discrete-time linear dynamics, according to

$$
\left\{\begin{array}{l}
x_{i}^{+}=A x_{i}+B_{2} u_{i}+B_{1} \omega_{i}, \quad i=1, \ldots, N \\
y_{i}=C x_{i}
\end{array}\right.
$$

where $A \in \mathbb{R}^{n \times n}, B_{2} \in \mathbb{R}^{n \times l}, B_{1} \in \mathbb{R}^{n \times h}, C \in \mathbb{R}^{m \times n}, x_{i} \triangleq x_{i}(k) \in \mathbb{R}^{n}$ and $x_{i}^{+} \triangleq x_{i}(k+1) \in \mathbb{R}^{n}$ are respectively the agent state at the current step $k$, and at the next step $k+1, u_{i} \triangleq u_{i}(k) \in \mathbb{R}^{l}$ is the agent control, $\omega_{i} \triangleq \omega_{i}(k) \in \mathbb{R}^{h}$ its disturbance, and $y_{i} \triangleq y_{i}(k) \in \mathbb{R}^{m}$ is the measured output and the variable on which agreement among the agents is sought. Moreover we require the system to satisfy $l \geq m$, i.e. to have a greater or equal number of inputs with respect to its outputs. For the sake of leaderless consensus, a priori we do not require $A$ to be Schur stable. Indeed, as shown in [7], $A$ has a role in determining the consensus function to which the agents converge under proper control. Here it can be thought to be assigned by a previous control design step. The agents can communicate on an undirected connected graph whose Laplacian matrix $\mathcal{L}$ has positive minimum nonzero and maximum eigenvalues respectively equal to $\underline{\lambda}_{\mathcal{L}}$, and $\bar{\lambda}_{\mathcal{L}}$. Thus, we can address the problem of finding a distributed control law for $u_{i}$ such that $\left\|y_{i} / \chi_{i}-y_{j} / \chi_{j}\right\|$ is minimized for $i, j=1, \ldots, N$ with respect to the disturbance $\omega \triangleq \operatorname{col}\left(\omega_{1}, \ldots, \omega_{N}\right)$, and where weight $\chi_{i} \in \mathbb{R}^{+}, i=1, \ldots, N$, i.e. for the sake of simplicity of analysis we associate the same scalar weight to the whole controlled output vector. If error $y_{i} / \chi_{i}-y_{j} / \chi_{j}=0, i, j=1, \ldots, N$, then we say that weighted consensus is achieved. By naming $D \triangleq \operatorname{diag}\left(1 / \chi_{1}, \ldots, 1 / \chi_{N}\right)$, we additionally define matrix $\hat{\mathcal{L}} \triangleq D \mathcal{L}$, which satisfies Lemma 3 in Appendix 1 , and whose positive minimum nonzero and maximum eigenvalues are respectively $\underline{\lambda}_{\hat{\mathcal{L}}}$, and $\bar{\lambda}_{\hat{\mathcal{L}}}$. In this work we focus on local controllers of the form

$$
\left\{\begin{array}{l}
x_{c_{i}}^{+}=A_{c} x_{c_{i}}+B_{c} s_{i}, \quad i=1, \ldots, N \\
u_{i}=C_{c} x_{c_{i}}+D_{c} s_{i}
\end{array}\right.
$$


where $x_{c_{i}} \triangleq x_{c_{i}}(k) \in \mathbb{R}^{2 l}$ is the agent controller state, and

$$
\begin{aligned}
A_{c} & =\left[\begin{array}{cc}
I_{l} & I_{l} \\
0_{l \times l} & 0_{l \times l}
\end{array}\right]_{2 l \times 2 l} \quad B_{c}=\left[\begin{array}{c}
\left(K_{i}-K_{d}\right) \\
K_{d}
\end{array}\right]_{2 l \times m} \\
C_{c} & =\left[\begin{array}{ll}
I_{l} & 0_{l \times l}
\end{array}\right]_{l \times 2 l} \quad D_{c}=\left[\left(K_{p}+K_{i}+K_{d}\right)\right]_{l \times m}
\end{aligned}
$$

where $K_{p}=\left[k_{p, i j}\right], K_{i}=\left[k_{i, i j}\right], K_{d}=\left[k_{d, i j}\right] \in \mathbb{R}^{l \times m}$ are gain matrices to be tuned, and where $s_{i} \triangleq s_{i}(k) \in \mathbb{R}^{m}$ is defined as

$$
s_{i} \triangleq \sum_{j=1}^{N} a_{i j}\left(\frac{y_{i}}{\chi_{i}}-\frac{y_{j}}{\chi_{j}}\right)
$$

Thus the closed-loop system for agent $i$ has dimension $\bar{n} \triangleq n+2 l$. As shown by [24], system (2) is a state representation of the discrete-time PID MIMO controller, whose $z$-transform between $s_{i}$ and $u_{i}$ is the transfer matrix $K_{p}+$ $K_{i} \frac{z}{z-1}+K_{d} \frac{z-1}{z}$, where its generic element at position $(i, j)$ is a PID whose gains are $k_{p, i j}, k_{i, i j}, k_{d, i j}$. The problem can be now restated as the one of finding matrices $B_{c}$, and $D_{c}$ such that the effect of disturbance $\omega$ on the weighted consensus is minimized.

\subsection{Fast Weighted Consensus}

In our previous work of [18], we provided two possible ways to tune the distributed PID controller, the first of which being based on imposing a given $\mathcal{H}_{\infty}$ constraint via LMIs on the closed-loop multi-agent system in order to minimize the additive disturbance effect on weighted consensus. In this work though we only focus on the second proposed possible way of tuning, which is concerned with achieving a multi-agent system fast response with respect to exogenous signals, such as disturbances, to reach weighted consensus. The two tuning techniques are quite similar, and the reader may refer to the mentioned reference for further details on $\mathcal{H}_{\infty}$ consensus design.

Let us introduce the following

Definition 1. System (1) is said to achieve fast weighted consensus with performance index $\tau \in \mathbb{R}^{+}$if for $\omega=\mathbf{0}$, and any initial condition, $\lim _{k \rightarrow \infty} \| y_{i} / \chi_{i}-$ $y_{j} / \chi_{j} \|=0$ for $i, j=1, \ldots, N$, and $\left(1-e^{-1}\right) \%$ of consensus is achieved in a maximum number of steps equal to $\lceil\tau\rceil$.

Note that the same kind of definition can be considered for sampled-data systems, by saying that system (1) achieves fast weighted consensus with a time constant inferior to $\tau T_{s}$, where $T_{s}$ is the system sampling time.

The following result is based on Theorem 4 of [24] shown in the Appendix 2.

Theorem 1. Given the system described by (1), where $N$ agents can communicate on an undirected connected graph; consider the distributed protocol of equations (2),(3),(4); then the systems achieve fast weighted consensus with performance index $\tau=-1 / \log (\psi)$, where $\psi \in \mathbb{R}: 0 \leq \psi<1$, if there exist two symmetric 
positive definite matrices $\underline{P}, \bar{P} \in \mathbb{R}^{\bar{n} \times \bar{n}}$ such that the LMI conditions of Theorem 4 are simultaneously satisfied for two LTI systems whose dynamic, input, and output matrices are respectively $\left(A, B_{2}, \underline{\lambda}_{\hat{\mathcal{L}}} C\right)$, and $\left(A, B_{2}, \bar{\lambda}_{\hat{\mathcal{L}}} C\right)$, and where the real constants $(a, b)$ to be set in Theorem 4 are chosen to be $(a, b)=(0, \psi)$.

Proof. In the sequel, the cited lemmas are shown in Appendix 1. The closed-loop dynamics for the generic agent $i$, by using (1),(2), and by defining the augmented state $\xi_{i} \triangleq \operatorname{col}\left(x_{i}, x_{c_{i}}\right) \in \mathbb{R}^{\bar{n}}$, and matrices $\bar{C} \triangleq\left[\begin{array}{ll}C & 0_{m \times 2 l}\end{array}\right], \tilde{B} \triangleq\left[\begin{array}{ll}B_{1}^{\top} & 0_{h \times(2 l)}\end{array}\right]^{\top}$ is given by

$$
\left\{\begin{array}{l}
\xi_{i}^{+}=\hat{A} \xi_{i}+\hat{B} \sum_{j=1}^{N} a_{i j}\left(\frac{\xi_{i}}{\chi_{i}}-\frac{\xi_{j}}{\chi_{j}}\right)+\tilde{B} \omega_{i} \\
y_{i}=\bar{C} \xi_{i}
\end{array}\right.
$$

where

$$
\hat{A}=\left[\begin{array}{cc}
A & B_{2} C_{c} \\
0 & A_{c}
\end{array}\right], \quad \hat{B}=\left[\begin{array}{c}
B_{2} D_{c} \bar{C} \\
B_{c} \bar{C}
\end{array}\right]
$$

By naming $\xi \triangleq \operatorname{col}\left(\xi_{1}, \ldots, \xi_{N}\right), y \triangleq \operatorname{col}\left(y_{1}, \ldots, y_{N}\right)$, gathering together the closed-loop agents dynamic, and performing the change of coordinates $\bar{\xi}=$ $\left(D \otimes I_{\bar{n}}\right) \xi$, it yields

$$
\left\{\begin{array}{l}
\bar{\xi}^{+}=\left(I_{N} \otimes \hat{A}+D \mathcal{L} \otimes \hat{B}\right) \bar{\xi}+\left(I_{N} \otimes \tilde{B}\right) \bar{\omega} \\
\bar{y}=\left(I_{N} \otimes \bar{C}\right) \bar{\xi}
\end{array}\right.
$$

where we named $\bar{\omega} \triangleq\left(D \otimes I_{h}\right) \omega, \bar{y} \triangleq\left(D \otimes I_{m}\right) y$, and we used point $(i)$ of Lemma 6. Similar to $[13,11]$, we define $\zeta_{i} \triangleq \bar{y}_{i}-\frac{1}{N} \sum_{j=1}^{N} \bar{y}_{j}$, and $\delta_{i} \triangleq \bar{\xi}_{i}-\frac{1}{N} \sum_{j=1}^{N} \bar{\xi}_{j}$. Thus $\zeta_{i}=\bar{C} \delta_{i}$. Note that if $\zeta_{i}=0$ for $i=1, \ldots, N$ then $\bar{y}_{i}=\bar{y}_{j}$, i.e. weighted consensus is achieved. If we now name $\delta \triangleq \operatorname{col}\left(\delta_{1}, \ldots, \delta_{N}\right)$, and $\zeta \triangleq \operatorname{col}\left(\zeta_{1}, \ldots, \zeta_{N}\right)$, we have that $\zeta=\left(I_{N} \otimes \bar{C}\right) \delta$, and $\delta=\bar{\xi}-\mathbf{1} \otimes \frac{1}{N} \sum_{j=1}^{N} \bar{\xi}_{j}=\left(\overline{\mathcal{L}} \otimes I_{\bar{n}}\right) \bar{\xi}$, where $\overline{\mathcal{L}}$ satisfies the conditions of Lemma 2. Thus $\zeta$ and $\bar{\xi}$ variables are linked by relationship $\zeta=\left(I_{N} \otimes \bar{C}\right)\left(\overline{\mathcal{L}} \otimes I_{\bar{n}}\right) \bar{\xi}=(\overline{\mathcal{L}} \otimes \bar{C}) \bar{\xi}$. Considering the change of coordinates $\delta=\left(\overline{\mathcal{L}} \otimes I_{\bar{n}}\right) \bar{\xi}$ for system (6), it yields

$$
\begin{aligned}
\delta^{+} & =\left(\overline{\mathcal{L}} \otimes I_{\bar{n}}\right)\left(I_{N} \otimes \hat{A}+\hat{\mathcal{L}} \otimes \hat{B}\right) \bar{\xi}+\left(\overline{\mathcal{L}} \otimes I_{\bar{n}}\right)\left(I_{N} \otimes \tilde{B}\right) \bar{\omega} \\
& =(\overline{\mathcal{L}} \otimes \hat{A}+\overline{\mathcal{L}} \hat{\mathcal{L}} \otimes \hat{B})\left(\delta+\mathbf{1} \otimes \frac{1}{N} \sum_{k=1}^{N} \bar{\xi}_{k}\right)+(\overline{\mathcal{L}} \otimes \tilde{B}) \bar{\omega} \\
& =(\overline{\mathcal{L}} \otimes \hat{A}+\overline{\mathcal{L}} \hat{\mathcal{L}} \otimes \hat{B}) \delta+(\overline{\mathcal{L}} \otimes \tilde{B}) \bar{\omega}
\end{aligned}
$$

where we used points $(i)$ of Lemma 2,3, and 6. According to the $(i i)$ point of Lemma 2, we employ the orthogonal matrix $U \in \mathbb{R}^{N \times N}$ to define the change of 
coordinates: $\hat{\delta} \triangleq\left(U^{\top} \otimes I_{\bar{n}}\right) \delta, \hat{\omega} \triangleq\left(U^{\top} \otimes I_{h}\right) \bar{\omega}, \hat{\zeta} \triangleq\left(U^{\top} \otimes I_{m}\right) \zeta$, so that the system equations in the new coordinates are given by

$$
\left\{\begin{aligned}
\hat{\delta}^{+}= & \left(U^{\top} \otimes I_{\bar{n}}\right)(\overline{\mathcal{L}} \otimes \hat{A}+\overline{\mathcal{L}} \hat{\mathcal{L}} \otimes \hat{B})\left(U \otimes I_{\bar{n}}\right) \hat{\delta} \\
& +\left(U^{\top} \otimes I_{\bar{n}}\right)(\overline{\mathcal{L}} \otimes \tilde{B}) \bar{\omega} \\
= & \left(\bar{\Lambda} \otimes \hat{A}+\bar{\Lambda} U^{\top} \hat{\mathcal{L}} U \otimes \hat{B}\right) \hat{\delta}+(\bar{\Lambda} \otimes \tilde{B}) \hat{\omega} \\
\hat{\zeta} \quad= & \left(U^{\top} \otimes I_{m}\right)\left(I_{N} \otimes \bar{C}\right)\left(U \otimes I_{\bar{n}}\right) \hat{\delta}=\left(I_{N} \otimes \bar{C}\right) \hat{\delta}
\end{aligned}\right.
$$

As shown in Lemma 2 , and 3 , being the last row and column of $\bar{\Lambda}$ zeros, and the last column of $U^{\top} \hat{\mathcal{L}} U$ zero, we can split (7) in two by dividing the system variables as $\hat{\delta}=\operatorname{col}\left(\hat{\delta}_{1}, \hat{\delta}_{2}\right), \hat{\omega}=\operatorname{col}\left(\hat{\omega}_{1}, \hat{\omega}_{2}\right)$, and $\hat{\zeta}=\operatorname{col}\left(\hat{\zeta}_{1}, \hat{\zeta}_{2}\right)$. It follows that, to conclude on system stability, we can study the reduced order system described by

$$
\left\{\begin{array}{l}
\hat{\delta}_{1}^{+}=\left(I_{N-1} \otimes \hat{A}+\hat{\mathcal{L}}_{1} \otimes \hat{B}\right) \hat{\delta}_{1}+\left(I_{N-1} \otimes \tilde{B}\right) \hat{\omega}_{1} \\
\hat{\zeta}_{1}=\left(I_{N-1} \otimes \bar{C}\right) \hat{\delta}_{1}
\end{array}\right.
$$

From Lemma 3, it exists an invertible matrix $V \in \mathbb{R}^{(N-1) \times(N-1)}: V^{-1} \hat{\mathcal{L}}_{1} V \triangleq$ $\Lambda=\operatorname{diag}\left(\lambda_{1}, \ldots, \lambda_{N-1}\right)$, where $0<\underline{\lambda}_{\hat{\mathcal{L}}} \leq \lambda_{i} \leq \bar{\lambda}_{\hat{\mathcal{L}}}$ for $i=1, \ldots, N-1$. Thus we can define a further change of coordinates, such that $\tilde{\delta}_{1} \triangleq\left(V^{-1} \otimes I_{\bar{n}}\right) \hat{\delta}_{1}$, $\tilde{\omega}_{1} \triangleq\left(V^{-1} \otimes I_{h}\right) \hat{\omega}_{1}$, and $\tilde{\zeta}_{1} \triangleq\left(V^{-1} \otimes I_{m}\right) \hat{\zeta}_{1}$. The latter yields

$$
\left\{\begin{array}{l}
\tilde{\delta}_{1}^{+}=\left(I_{N-1} \otimes \hat{A}+\Lambda \otimes \hat{B}\right) \bar{\delta}_{1}+\left(I_{N-1} \otimes \tilde{B}\right) \tilde{\omega}_{1} \\
\tilde{\zeta}_{1}=\left(I_{N-1} \otimes \bar{C}\right) \tilde{\delta}_{1}
\end{array}\right.
$$

We can now separate (8) in $N-1$ subsystems, each of them being governed by

$$
\left\{\begin{array}{l}
\tilde{\delta}_{1_{i}}^{+}=\left[\begin{array}{c}
\tilde{x}_{1_{i}}^{+} \\
\tilde{x}_{1, c_{i}}^{+}
\end{array}\right]=\left[\begin{array}{cc}
\left(A+B_{2} D_{c}\left(\lambda_{i} C\right)\right) & B_{2} C_{c} \\
B_{c}\left(\lambda_{i} C\right) & A_{c}
\end{array}\right]\left[\begin{array}{c}
\tilde{x}_{1_{i}} \\
\tilde{x}_{1, c_{i}}
\end{array}\right]+\left[\begin{array}{c}
B_{1} \\
0
\end{array}\right] \tilde{\omega}_{1_{i}} \\
\tilde{\zeta}_{1_{i}}=C \tilde{x}_{1_{i}}
\end{array}\right.
$$

System (9) can be equivalently seen as the closed-loop form of the two following systems

$$
\left\{\begin{array}{l}
\tilde{x}_{1_{i}}^{+}=A \tilde{x}_{1_{i}}+B_{2} \tilde{u}_{i}+B_{1} \tilde{\omega}_{1_{i}} \\
\tilde{y}_{1_{i}} \triangleq\left(\lambda_{i} C\right) \tilde{x}_{1_{i}} \\
\tilde{\zeta}_{1_{i}}=C \tilde{x}_{1_{i}}
\end{array}, \quad\left\{\begin{array}{l}
\tilde{x}_{1, c_{i}}^{+}=A_{c} \tilde{x}_{1, c_{i}}+B_{c} \tilde{y}_{1_{i}} \\
\tilde{u}_{i} \triangleq C_{c} \tilde{x}_{1, c_{i}}+D_{c} \tilde{y}_{1_{i}}
\end{array}\right.\right.
$$

where $\tilde{y}_{1_{i}}$, and $\tilde{\zeta}_{1_{i}}$ are respectively the measured and controlled output variables of the controlled system. Thus, we can reformulate the problem as the one finding matrices $B_{c}$, and $D_{c}$ such that for $i=1, \ldots, N-1$ the closed-loop system of (10) is Schur stable when $\omega_{1_{i}}=0$. Moreover, since we are interested in speeding up consensus reaching with respect to exogenous signals, we want to push the overall closed-loop system eigenvalues closed to zero as much as possible. For 


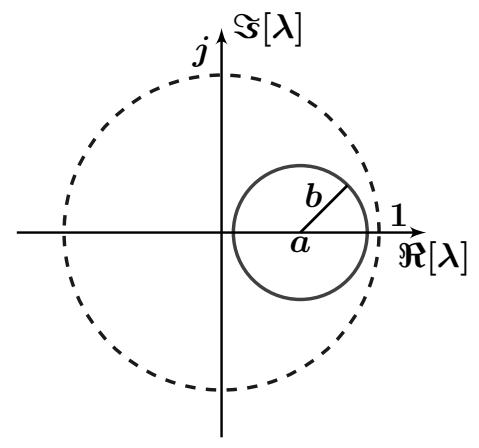

Fig. 1. $\mathcal{F}_{D}$ region in the complex plane defined via parameters $(a, b)$.

this purpose we invoke Theorem 4, whose results can be directly applied to one generic system of the form of (10). Here it is shown that, given two constants $a \in \mathbb{R}$, and $b \in \mathbb{R}_{0}^{+}$, if there exists a symmetric positive definite matrix $P_{i}$ such that the given LMI condition in the theorem is satisfied, then system (10) is stable with all its eigenvalues laying in the complex plane region defined by

$$
\mathcal{F}_{D} \triangleq\left\{(\Re[\lambda], \Im[\lambda]):(\Re[\lambda]+a)^{2}+\Im[\lambda]^{2}<b^{2}\right\}
$$

where $\lambda$ is the complex variable, (see Fig. 1). It is important to stress that the mentioned LMI conditions are affine in the system matrices, variables and matrix $P_{i}$. We make use of this fact to provide sufficient conditions for which it exists a controller of the considered form such that the mentioned LMI is simultaneously verified for $i=1, \ldots, N-1$. Since the generic eigenvalue of $\hat{\mathcal{L}}_{1}: \lambda_{i}$ is such that $\underline{\lambda}_{\hat{\mathcal{L}}} \leq \lambda_{i} \leq \bar{\lambda}_{\hat{\mathcal{L}}}$, then it always exists $\alpha_{i} \in \mathbb{R}: 0 \leq \alpha_{i} \leq 1$ so that $\lambda_{i}=\alpha_{i} \underline{\lambda}_{\hat{\mathcal{L}}}+\left(1-\alpha_{i}\right) \bar{\lambda}_{\hat{\mathcal{L}}}$. Notice that the systems to be stabilized, appearing in the first set of equation in (10), can be seen as one single system with an uncertain measurement matrix, whose parameter is $\lambda_{i}$. In other words, $C_{i} \triangleq \lambda_{i} C$, and $\exists \alpha_{i}: C_{i}=\alpha_{i} C_{\text {low }}+\left(1-\alpha_{i}\right) C_{\text {up }}$, where $C_{\text {low }} \triangleq \underline{\lambda}_{\hat{\mathcal{L}}} C$, and $C_{\text {up }} \triangleq \bar{\lambda}_{\hat{\mathcal{L}}} C$, i.e. it can be written as a convex combination of the extreme matrices $C_{\text {low }}$, and $C_{u p}$. Thus, as in [11], we make use of classic results of robust linear control, and in particular by introducing an affine parameter dependent Lyapunov matrix $P\left(\alpha_{i}\right) \triangleq \alpha_{i} \underline{P}+$ $\left(1-\alpha_{i}\right) \bar{P}$, where $\underline{P}, \bar{P}$ are Lyapunov matrices solution of simultaneous LMI of Theorem 4 written for respectively $C_{\text {low }}$, and $C_{u p}$. Thus, it is easy to show that if $\underline{P}, \bar{P}$ exist, then the controller solves the problem $\forall \lambda \in \mathbb{R}: \underline{\lambda}_{\hat{\mathcal{L}}} \leq \lambda \leq \bar{\lambda}_{\hat{\mathcal{L}}}$, and in particular for $\lambda=\lambda_{i}$, for $i=1, \ldots, N-1$. Such a controller is easily found from the solution of the aforementioned LMI condition. Indeed among the LMI variables there are matrices $B_{c}$, and $D_{c}$, from which it is easy to deduce the PID gain matrices $K_{p}, K_{i}$, and $K_{d}$ by employing relations in (3). Eventually, in order to place the closed-loop system eigenvalues closed to 0 , we set $a=0$, and $b=\psi$, where $\psi: 0 \leq \psi<1$. Thus, all system eigenvalues are guaranteed to have a module inferior to $\psi$. As a result, the system has the slowest time-constant 
inferior to $-T_{s} / \log (\psi)$. In terms of number of iterations, such performance is equal to a maximum value $\lceil-1 / \log (\psi)\rceil$ of iterations.

Remark 1. If the mentioned LMI has a solution, then the closed-loop multi-agent system is guaranteed to be stable. In addition, having employed a PID structure for the distributed controller may suggest that consensus should be reached for any constant disturbance vector $\omega$. Unfortunately, this is not automatically guaranteed in the MIMO case by the mentioned LMI conditions, and in this framework it is only verified a posteriori. Note that the MIMO PID controller is not block diagonal. Nonetheless, if such LMI has a solution then, according to the well-known Francis equations, a necessary condition for the proposed controller to reject constant exogenous signals is that $l \geq m$.

Remark 2. If the mentioned LMI has a solution and consensus is reached, still the disturbance has a role in determining the common function to which the agents converge, called consensus function.

\section{Leader-follower Consensus with Time-varying Reference State}

\subsection{Problem Formulation}

The results shown in Section 2 can be easily applied to solve the following leaderfollower problem. Consider $N+1$ discrete-time linear agents, whose dynamics are described by

$$
\left\{\begin{array}{l}
x_{0}^{+}=A x_{0}+B_{1} u_{0} \\
y_{0}=C x_{0}
\end{array}, \quad\left\{\begin{array}{l}
x_{i}^{+}=A x_{i}+B_{2} u_{i}, \quad i=1, \ldots, N \\
y_{i}=C x_{i}
\end{array}\right.\right.
$$

where $A \in \mathbb{R}^{n \times n}, B_{1} \in \mathbb{R}^{n \times h}, B_{2} \in \mathbb{R}^{n \times l}, C \in \mathbb{R}^{m \times n}, x_{0} \triangleq x_{0}(k) \in \mathbb{R}^{n}$ is the state of the $N+1$ agent, called leader, $y_{0} \triangleq y_{0}(k) \in \mathbb{R}^{m}$ is its measured output and the variable on which we want the follower measured and controlled outputs $y_{i}$ to converge, and $u_{0} \triangleq u_{0}(k) \in \mathbb{R}^{h}$ is a time-varying unknown control acting on the leader dynamics. We additionally suppose that $l \geq m$. Concerning the remaining $N$ follower agents, system description equivalent to (1) holds. The followers are assumed to communicate on an undirected connected graph whose Laplacian matrix is $\mathcal{L}$. The leader can pass information to a subset of followers. If agent $i$ receives information from the leader, then we set $a_{i 0}$ to 1 , and 0 otherwise. Thus we can define $\mathcal{M} \triangleq \mathcal{L}+\operatorname{diag}\left(a_{10}, \ldots, a_{N 0}\right)$, which is symmetric and positive definite. Differently from the leaderless consensus case, without loss of generality we consider $A$ to be Schur stable. The aim of the present problem is indeed not the one of stabilizing each single agent, but rather to steer the follower agents state to the leader one despite the presence of $u_{0}$, which makes the leader dynamics time-varying. Moreover, as done for the leaderless case, we consider the general case of weighted consensus. In other words we aim at finding a distributed control law to minimize $\left\|y_{i} / \chi_{i}-y_{0} / \chi_{0}\right\|$ for $i=1, \ldots, N$, 
where $\chi_{i}, \chi_{0} \in \mathbb{R}^{+}$. In order to accomplish such objective we aim to employ the controller of form (2), (3), where we consider a modified variable $s_{i}$ to take into account the communication with the leader agent, according to

$$
s_{i}=\sum_{j=1}^{N} a_{i j}\left(\frac{y_{i}}{\chi_{i}}-\frac{y_{j}}{\chi_{j}}\right)+a_{i 0}\left(\frac{y_{i}}{\chi_{i}}-\frac{y_{0}}{\chi_{0}}\right)
$$

Eventually, by using $D$, which we recall to be $D=\operatorname{diag}\left(1 / \chi_{1}, \ldots, 1 / \chi_{N}\right)$, we can additionally define $\hat{\mathcal{M}} \triangleq D \mathcal{M}$, which satisfies Lemmas 4 , and 5 , and it has minimum and maximum positive real eigenvalues equal to $\underline{\lambda}_{\hat{\mathcal{M}}}$, and $\bar{\lambda}_{\hat{\mathcal{M}}}$ respectively.

\subsection{Fast Weighted Leader-follower Consensus}

Similar to Definition 1, we provide the following

Definition 2. System (11) is said to achieve fast weighted leader-follower consensus with performance index $\tau \in \mathbb{R}^{+}$if for any initial condition, $\lim _{k \rightarrow \infty} \| y_{i} / \chi_{i}$ $y_{0} / \chi_{0} \|=0$ for $i=1, \ldots, N$, when $u_{0}=0$, and $\left(1-e^{-1}\right) \%$ of consensus is achieved in a maximum number of steps equal to $\lceil\tau\rceil$.

The following result, similar to Theorem 1, is based on Theorem 4 of [24] in the Appendix 1.

Theorem 2. Given the system described by (11), where $N$ follower agents can communicate on an undirected connected graph, and one leader can communicate with a non-empty subset of followers; consider the distributed protocol of equations (2),(3),(12); then the systems achieve fast leader-follower consensus with performance index $\tau=-1 / \log (\psi)$, where $\psi \in \mathbb{R}: 0 \leq \psi<1$, if there exist two symmetric positive definite matrices $\underline{P}, \bar{P} \in \mathbb{R}^{\bar{n} \times \bar{n}}$ such that the LMI conditions of Theorem 4 are simultaneously satisfied for two LTI systems whose dynamic, input and output matrices are respectively $\left(A, B_{2}, \underline{\lambda}_{\hat{\mathcal{M}}} C\right)$, and $\left(A, B_{2}, \bar{\lambda}_{\hat{\mathcal{M}}} C\right)$, and where the real constants $(a, b)$ to be set in Theorem 4 are chosen to be $(a, b)=(0, \psi)$.

Proof. The proof is similar to the one of Theorem 1. By defining error $e_{i} \triangleq$ $x_{i}-x_{0} \chi_{i} / \chi_{0}, \xi_{i} \triangleq \operatorname{col}\left(e_{i}, x_{c_{i}}\right)$, and $\zeta_{i} \triangleq C e_{i}$ the closed-loop system for the generic follower agent $i$ is given by

$$
\left\{\begin{array}{l}
\xi_{i}^{+}=\hat{A} \xi_{i}+\hat{B}\left(\sum_{j=1}^{N} a_{i j}\left(\frac{\xi_{i}}{\chi_{i}}-\frac{\xi_{j}}{\chi_{j}}\right)+a_{i 0} \frac{\xi_{i}}{\chi_{i}}\right)+\chi_{i} \tilde{B} u_{0} \\
\zeta_{i}=\bar{C} \xi_{i}
\end{array}\right.
$$

where $\hat{A}, \hat{B}, \bar{C}$ are defined in (5), and $\tilde{B} \triangleq\left[\begin{array}{ll}-B_{1}^{\top} / \chi_{0} & 0_{h \times 2 l}\end{array}\right]^{\top}$. Defining $\boldsymbol{u}_{\mathbf{0}} \triangleq$ $\mathbf{1}_{N} \otimes u_{0}$, and $\boldsymbol{u}_{\mathbf{0}} \triangleq\left(D \otimes I_{\bar{n}}\right) \tilde{\boldsymbol{u}}_{\mathbf{0}}$, we then gather the $N$ agent equations together

$$
\left\{\begin{array}{l}
\xi^{+}=\left(I_{N} \otimes \hat{A}+\mathcal{M} D \otimes \hat{B}\right) \xi+\left(I_{N} \otimes \tilde{B}\right) \tilde{\boldsymbol{u}}_{\mathbf{0}} \\
\zeta=\left(I_{N} \otimes \bar{C}\right) \xi
\end{array}\right.
$$


We consider the change of coordinates $\bar{\xi} \triangleq\left(D \otimes I_{\bar{n}}\right) \xi$, and define $\overline{\boldsymbol{u}}_{\mathbf{0}} \triangleq\left(D \otimes I_{\bar{n}}\right) \tilde{\boldsymbol{u}}_{\mathbf{0}}$, $\bar{\zeta} \triangleq\left(D \otimes I_{\bar{n}}\right) \zeta$, system (13) can be rewritten in the new coordinates as

$$
\left\{\begin{array}{l}
\bar{\xi}^{+}=\left(I_{N} \otimes \hat{A}+\hat{\mathcal{M}} \otimes \hat{B}\right) \bar{\xi}+\left(I_{N} \otimes \tilde{B}\right) \overline{\boldsymbol{u}}_{\mathbf{0}} \\
\bar{\zeta}=\left(I_{N} \otimes \bar{C}\right) \bar{\xi}
\end{array}\right.
$$

From the definition of $\hat{\mathcal{M}}$, there exists an orthogonal matrix $U: U^{\top} \hat{\mathcal{M}} U \triangleq \Lambda=$ $\operatorname{diag}\left(\lambda_{1}, \ldots, \lambda_{N}\right)$, where $\lambda_{i} \in \mathbb{R}: \lambda_{i}>0$ for $i=1, \ldots, N$, so that we can define the change of coordinates $\bar{\xi} \triangleq\left(U \otimes I_{\bar{n}}\right) \hat{\xi}, \tilde{\boldsymbol{u}}_{\mathbf{0}} \triangleq\left(U \otimes I_{h}\right) \hat{\boldsymbol{u}}_{\mathbf{0}}, \bar{\zeta} \triangleq\left(U \otimes I_{m}\right) \hat{\zeta}$. By applying similar calculation as in the previous sections, the global system in the new coordinates is

$$
\left\{\begin{array}{l}
\hat{\xi}^{+}=\left(I_{N} \otimes \hat{A}+\Lambda \otimes \hat{B}\right) \hat{\xi}+\left(I_{N} \otimes \tilde{B}\right) \hat{\boldsymbol{u}}_{\mathbf{0}} \\
\hat{\zeta}=\left(I_{N} \otimes \bar{C}\right) \hat{\xi}
\end{array}\right.
$$

Splitting (14) in $N$ subsystems yields the following equation for subsystem $i$

$$
\left\{\begin{array}{l}
\hat{\xi}_{i}^{+}=\left[\begin{array}{cc}
\left(A+B_{2} D_{c}\left(\lambda_{i} C\right)\right) & B_{2} C_{c} \\
B_{c}\left(\lambda_{i} C\right) & A_{c}
\end{array}\right] \hat{\xi}_{i}+\left[\begin{array}{c}
-B_{1} / \chi_{0} \\
0
\end{array}\right] \hat{u}_{0} \\
\hat{\zeta}_{i}=C \hat{e}_{i}
\end{array}\right.
$$

where $\hat{\xi}_{i} \triangleq \operatorname{col}\left(\hat{e}_{i}, \hat{x}_{c_{i}}\right)$. System (15) can be equivalently described as the connection of the two following systems

$$
\left\{\begin{array}{l}
\hat{e}_{i}^{+}=A \hat{e}_{i}+B_{2} \hat{u}_{i}-B_{1} / \chi_{0} \hat{u}_{0} \\
\hat{y}_{i} \triangleq\left(\lambda_{i} C\right) \hat{e}_{i} \\
\hat{\zeta}_{i}=C \hat{e}_{i}
\end{array} \quad, \quad\left\{\begin{array}{l}
\hat{x}_{c_{i}}^{+}=A_{c} \hat{x}_{c_{i}}+B_{c} \hat{y}_{i} \\
\hat{u}_{i} \triangleq C_{c} \hat{x}_{c_{i}}+D_{c} \hat{y}_{i}
\end{array}\right.\right.
$$

The rest of the proof is similar to the last part of the one of Theorem 1. In particular, since system (15) can be seen as one system with uncertain parameter $\lambda_{i} \in\left[\underline{\lambda}_{\hat{\mathcal{M}}}, \bar{\lambda}_{\hat{\mathcal{M}}}\right]$, we make use of LMI conditions of Theorem 4 , and we impose them to be simultaneously satisfied for two systems at the vertexes of the polytope having matrices $\left(A, B_{2}, \underline{\lambda}_{\hat{\mathcal{M}}} C\right)$, and $\left(A, B_{2}, \bar{\lambda}_{\hat{\mathcal{M}}} C\right)$. The proof is concluded as for Theorem 1.

Remark 3. A similar remark to the one of Remark 1 holds for the leader-follower consensus case too. In particular, having imposed a PID structure for the distributed controller is not sufficient to guarantee rejection of constant $u_{0}$ vectors in the MIMO case. A necessary condition though is given by $l \geq m$.

\section{Wind Farm Control Problem}

\subsection{Wind Turbine Model}

The wind turbine model describes the conversion from wind power to electric power. The wind kinetic energy captured by the turbine is turned into mechanical 


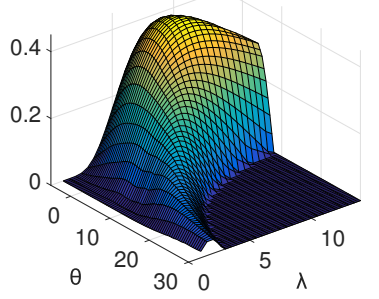

(a) CART power coefficient.

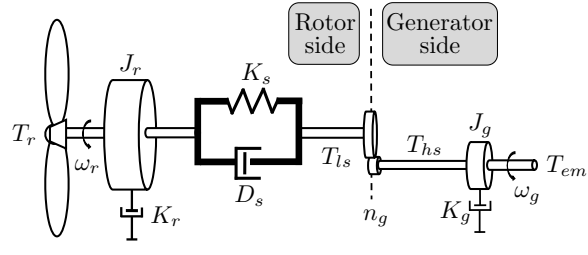

(b) Two-mass model of WT mechanics.

Fig. 2. Wind turbine aerodynamics and mechanics.

energy of the turbine rotor, turning at an angular speed $\omega_{r}$ and subject to a torque $T_{r}$. In terms of extracted power, it can be described by the nonlinear function $P_{r}=\omega_{r} T_{r}=1 / 2 \rho \pi R^{2} v^{3} C_{p}(\lambda, \vartheta)$, where $\rho$ is the air density, $R$ is the radius of the rotor blades, $\vartheta$ is the pitch angle, $v$ is the effective wind speed representing the wind field impact on the turbine, $\lambda$ is the tip speed ratio given by $\lambda=\frac{\omega_{r} R}{v} . C_{p}$, nonlinear function of the tip speed ratio and pitch angle, is the power coefficient. This is typically provided in turbine specifications as a look-up table. As far as the turbine parameters are concerned, in this work we make use of the CART (Controls Advanced Research Turbine) power coefficient shown in Fig. 2a. This turbine is located at NREL's National Wind Technology Center. Nonetheless, we employ a polynomial approximation of the latter for the purpose of the synthesis of the controller. Referring to a two-mass model as in [25], and as shown in Fig. $2 \mathrm{~b}$, then, the low speed shaft torque $T_{l s}$ acts as a braking torque on the rotor, the generator is driven by the high speed torque $T_{h s}$, and braked by the generator electromagnetic torque $T_{e m}$. The drive train turns the slow rotor speed into high speed on the generator side, $\omega_{g}$. Finally $J_{r}$ is the rotor inertia, $K_{r}$, and $K_{g}$ damping coefficients, $n_{g}$ the gear ratio, and $J_{g}$ the generator inertia. The dynamics of the WT is thus described by $J_{r} \dot{\omega}_{r}=T_{r}-K_{r} \omega_{r}-T_{l s}$, and $J_{g} \dot{\omega}_{g}=T_{h s}-K_{g} \omega_{g}-T_{e m}$. In this paper we also consider a first order system to model the pitch actuator, endowed with a sigmoid function $\sigma: \mathbb{R} \rightarrow\left[\vartheta_{\min }, \vartheta_{\max }\right]$ to model the pitch saturation. In addition, for ease of further development we can bring the system equations back on the low speed side, obtaining the simplified overall model

$$
\left\{\begin{array}{l}
\tau_{\vartheta} \dot{\vartheta}_{s}=-\vartheta_{s}+\vartheta_{r} \\
\vartheta=\sigma\left(\vartheta_{s}\right) \\
J_{t} \dot{\omega}_{r}=\frac{P_{r}\left(\omega_{r}, \vartheta, v\right)}{\omega_{r}}-K_{t} \omega_{r}-T_{g}
\end{array}\right.
$$

where $T_{g} \triangleq n_{g} T_{e m}, J_{t} \triangleq J_{r}+n_{g}^{2} J_{g}, K_{t} \triangleq K_{r}+n_{g}^{2} K_{g}$, and where we used the relation $n_{g}=\omega_{g} / \omega_{r}=T_{l s} / T_{h s}$. Eventually, neglecting the generator losses, the electric power delivered to the grid is $P=T_{g} \omega_{r}$. The system inputs are $T_{g}$, and $\vartheta_{r}$, while the wind speed $v$ acts as a disturbance. The feasible domain of the state variable is $X \triangleq\left\{\left(\omega_{r}, \vartheta\right) \in \mathbb{R}^{2}: \omega_{r} \in\left[\omega_{r, \min }, \omega_{r, \max }\right], \vartheta \in\left[\vartheta_{\text {min }}, \vartheta_{\text {max }}\right]\right\}$. 


\subsection{Problem Statement}

In the sequel, for consistency of notation, we add the index $i$ to the WT variables described in the previous subsection when referring to turbine $i$ variables, and we drop it when the results hold for any WT. At low wind speed, WTs are usually operated according to the well-known MPPT algorithm. The maximum power that a WT can extract from the wind is thus attained for a constant value of $\vartheta$, named here $\vartheta^{\circ}$, depending on the turbine $C_{p}$, and by controlling the WT to track the optimal tip speed ratio value $\lambda^{o} \triangleq \arg \max _{\lambda} P_{r}\left(v, \vartheta^{o}, \lambda\right)=$ $\arg \max _{\lambda} C_{p}\left(\lambda, \vartheta^{o}\right)$. We name $C_{p}^{o} \triangleq C_{p}\left(\lambda^{o}, \vartheta^{o}\right)$, and $P^{o}(v) \triangleq P_{r}\left(v, \vartheta^{o}, \lambda^{o}\right)$. For the considered CART turbine $\lambda^{o} \cong 8$. Nonetheless, when considering the wake effect in the optimization step of a farm of $N$ WTs, the optimal value of $C_{p}$ related to the generic turbine $i$ is such that $C_{p, i}^{\star} \leq C_{p}^{o}$. As a matter of fact, this implies that a turbine $i$ should track an optimal power reference $P_{i}^{\star}\left(v_{i}\right)$ that satisfies $P_{i}^{\star}\left(v_{i}\right) \leq P_{i}^{o}\left(v_{i}\right)$, i.e. it has to be deloaded if maximum wind farm power is seek. The reader may refer to the works of e.g. $[26,19]$ to see how values $C_{p, i}^{\star}$ can be computed. According to the usually employed wake models, as well as the following

Assumption 1 For $i=1, \cdots, N$, $v_{i}$ is such that $P_{i}^{\star}\left(v_{i}\right)<P_{n}$, where $P_{n}$ is the nominal WT power.

Then, the static optimization step needs to be run only when the wind direction changes, as optimal values $C_{p, i}^{\star}$ do not depend on the wind speed value, [19].

Assumption 2 The average wind direction is considered to be slowly varying with respect to the system dynamics. Thus, it is considered to be constant.

We can formulate the overall WF control problem in two subproblems, the first of which being

Problem 1. Consider the system described by (17). Given an effective wind speed signal $v(t)$, and a time-varying reference trajectory $P^{r e f}(t)$, verifying $P^{r e f}(t) \leq$ $P^{o}(t) \forall t \geq 0$, find the signals $\left(\vartheta_{r}(t), T_{g}(t)\right) \forall t \geq 0$ such that $\lim _{t \rightarrow \infty} \mid P^{r e f}(t)-$ $P(t) \mid=0$ for every initial condition $\left(\omega_{r}(0), \vartheta(0)\right) \in X: P(0) \leq P^{o}(0)$.

Let us now assume that each local WT controller can measure, or estimate, the effective wind speed $v_{m, i}(t)$ such that $v_{i}(t)=v_{m, i}(t)+v_{d, i}(t) \forall t \geq 0$. Thus $v_{d, i}$ represents a nonmeasurable time varying disturbance for turbine $i$.

Assumption 3 We consider small zero-mean disturbances $v_{d, i}$ with respect to $v_{m, i}$, and slowly-varying with respect to the dynamics of (17).

Each WT can compute its power reference, as described in [27], from its maximum available power $P_{i}^{o}$, according to

$$
P_{i}^{f w} \triangleq P_{i}\left(v_{m, i}\right)=\frac{C_{p, i}^{\star}}{C_{p}^{o}} P_{i}^{o}\left(v_{m, i}\right)
$$


which is optimal in nominal conditions, i.e. $P_{i}^{\star}\left(v_{i}\right)=\frac{C_{p, i}^{\star}}{C_{p}^{o}} P_{i}^{o}\left(v_{i}\right)$ when $v_{d, i} \equiv 0$. We can additionally require the WTs to meet an optimal relative power sharing condition given by

$$
\frac{P_{i}}{\chi_{i}}=\frac{P_{k}}{\chi_{k}} \quad i, k=1, \cdots, N
$$

Indeed, by naming $P_{\infty}^{o}$ the maximum power that a WT could extract from the wind if there was no wake effect, from (18) we have that $P_{i} / C_{p, i}^{\star}=P_{i}^{o} / C_{p}^{o}=$ $\gamma_{i} P_{\infty}^{o} / C_{p}^{o}, i=1, \cdots, N$, thus $P_{i} / \gamma_{i} C_{p, i}^{\star}=P_{k} / \gamma_{k} C_{p, k}^{\star}, i, k=1, \cdots, N$. We name $\chi_{i} \triangleq \gamma_{i} C_{p, i}^{\star} \in \mathbb{R}^{+}$, and where $\gamma_{i}=P_{i}^{o} / P_{\infty}^{o}=\left(v_{i} / v_{\infty}\right)^{3}$ are constant values for any value of $v_{\infty}$ according to Assumptions 1 , and 2, being $v_{\infty}$ the free stream wind speed. In the sequel, in order to make the difference with condition (19) clear, we will refer to (18) as the absolute power reference. This is optimal if the corresponding $v_{d, i}=0$. Despite being redundant information with respect to (18) in nominal conditions, as it will be made clear in the sequel, condition (19) provides additional signals that can be exploited when the system is subject to disturbances. We can now state the second subproblem.

Problem 2. Given $N$ identical WTs, allowed to communicate on an undirected connected graph $\mathcal{G}$; given optimal values $C_{p, i}^{\star}$, and $\chi_{i}, i=1, \ldots, N$; find $P_{i}^{r e f}(t)$ $\forall t \geq 0, i=\ldots, N$ such that each $P_{i}$ tracks (18), while minimizing the error $\left|P_{i} / \chi_{i}-P_{j} / \chi_{j}\right|, i, j=\ldots, N$, under the presence of $v_{d, i}(t)$.

The idea behind Problem 2 is to exploit additional information concerning optimal WTs relative power values in order to even out the system disturbances from the optimal power sharing defined by the higher optimization step.

\section{$5 \quad$ Wind Farm Control Design}

\subsection{Wind Turbine Control for Deloaded Mode}

According to the WF optimization problem, it turns out that every WT causing a reduction of available wind power of another one, is very likely to be subject to an optimal $C_{p}$ value such that $C_{p, i}^{\star}<C_{p}^{o}$, i.e. strictly inferior. Thus, WTs whose $C_{p, i}^{\star}$ verifies $C_{p, i}^{\star}=C_{p}^{o}$ should simply perform classic MPPT regardless the disturbances of the system and the other WTs operating points, and they can be controlled with classic local controllers. In the sequel we only consider WTs that have to be strictly deloaded with respect to their $P_{i}^{o}$.

In this subsection we proposed an approximated asymptotic output tracking (AOT) technique to control a WT in deloaded mode, and it is based on the work of [25]. However, differently from [25], power tracking is here achieved by employing both the rotor angular speed and the pitch angle. The local WT controller is composed of a first loop to control $\omega_{r}$. We impose a first order dynamics to the rotor speed tracking error $\varepsilon_{\omega} \triangleq \omega^{r e f}-\omega_{r}$, i.e. $\dot{\varepsilon}_{\omega}+a_{0} \varepsilon_{\omega}=0$ by choosing $a_{0} \in \mathbb{R}^{+}$. If we name $w \triangleq a_{0} \omega^{r e f}+\dot{\omega}^{r e f}$, this is attained by using (17) as

$$
T_{e m}=T_{r}-\left(K_{t}-a_{0} J_{t}\right) \omega_{r}-J_{t} w
$$


We choose to regulate the power output $P$ by acting on the pitch angle, and by imposing a first order dynamics to the electric power tracking error $\varepsilon_{p} \triangleq$ $P^{r e f}-P$, i.e.

$$
\dot{\varepsilon}_{p}+b_{0} \varepsilon_{p}=0
$$

where $b_{0} \in \mathbb{R}^{+}$. This is attained via feedback linearization (FL) on (17) by choosing the feedback linearizing input

$$
\begin{aligned}
\vartheta_{r}= & \frac{1}{\beta\left(\omega_{r}, \vartheta_{s}, v\right)}\left(\dot{P}^{r e f}-\omega_{r} \frac{\partial T_{r}}{\partial v} \dot{v}+\frac{\omega_{r}}{\tau_{\vartheta}} \frac{\partial T_{r}}{\partial \vartheta} \frac{d \sigma}{d \vartheta_{s}} \vartheta_{s}+J_{t} \dot{w} \omega_{r}\right. \\
& \left.+\left(2\left(K_{t}-a_{0} J_{t}\right) \omega_{r}-T_{r}+J_{t} w-\omega_{r} \frac{\partial T_{r}}{\partial \omega_{r}}\right)\left(-a_{0} \omega_{r}+w\right)+b_{0} \varepsilon_{p}\right)
\end{aligned}
$$

where $\frac{\partial T_{r}}{\partial \omega_{r}}, \frac{\partial T_{r}}{\partial \vartheta}$, and $\frac{\partial T_{r}}{\partial v}$ are functions of $\left(\omega_{r}, \vartheta, v\right)$, and $\beta \triangleq \frac{\omega_{r}}{\tau_{\vartheta}} \frac{d \sigma}{d \vartheta_{s}} \frac{\partial T_{r}}{\partial \vartheta}$.

As pointed out in our previous work [28], there exist points in which $\beta=0$, called singular points, i.e. points in which (22), feedback linearizing input with respect to output $P$, is not well-defined. These points are determined by the solution of $\operatorname{dfrac} \partial C_{q} \partial \vartheta\left(\omega_{r}, \vartheta, v\right)=\frac{\partial C_{q}}{\partial \vartheta}(\lambda, \vartheta)=0$, being $C_{q} \triangleq \frac{C_{p}}{\lambda}$, since $\beta\left(\omega_{r}, \vartheta_{s}, v\right)=\frac{\omega_{r}}{2 \tau_{\vartheta}} \rho \pi R^{3} v^{2} \frac{d \sigma}{d \vartheta_{s}} \frac{\partial C_{q}}{\partial \vartheta}\left(\omega_{r}, \sigma\left(\vartheta_{s}\right), v\right) \cong \frac{\omega_{r}}{2 \tau_{\vartheta}} \rho \pi R^{3} v^{2} \frac{\partial C_{q}}{\partial \vartheta}\left(\omega_{r}, \vartheta, v\right)$ in the domain of interest of $\vartheta$, and $\omega_{r}, v>0$. In Fig. 3a the white area represents $\Lambda=\left\{(\lambda, \vartheta):\left(\omega_{r}, \vartheta\right) \in \mathcal{X} \wedge \beta<0\right\}$. If $\omega^{r e f}$ is chosen to let $\lambda$ be in a neighborhood of $\lambda^{\circ}$, and $\vartheta>\vartheta^{\circ}$ in order to deload the WT, where $\vartheta^{\circ} \simeq 0^{\circ}$ for CART turbine, then it is clear that $\beta$ is negative-valued in the points of functioning of interest. In order to ensure that the trajectories of the closed loop system, defined by (17), (20), (22), do not pass through singular points, we consider a modified FL function for $\vartheta_{r}$, by replacing the $\beta$ function appearing in (22) with

$$
\begin{gathered}
\hat{\beta} \triangleq \frac{\omega_{r}}{2 \tau_{\vartheta}} \rho \pi R^{3} v^{2} \frac{d \sigma}{d \vartheta_{s}}\left(\frac{\partial C_{q}}{\partial \vartheta}(\lambda, \vartheta)-\varepsilon(\lambda, \vartheta)\right) \\
\varepsilon(\lambda, \vartheta) \triangleq \begin{cases}\varrho \max \left\{\frac{\partial C_{q}}{\partial \vartheta}(\lambda, \vartheta), 0\right\} & \text { if } \frac{\partial C_{q}}{\partial \vartheta}(\lambda, \vartheta) \neq 0 \\
\epsilon_{1} & \text { otherwise }\end{cases}
\end{gathered}
$$

where $\epsilon_{1}$ is a small positive value, and $\varrho>1$ is a tunable parameter to let some margin to have $\hat{\beta}$ negative-valued in the system trajectories. Thus we obtain an expanded negative-valued area $\hat{\Lambda}=\left\{(\lambda, \vartheta):\left(\omega_{r}, \vartheta\right) \in \mathcal{X} \wedge \hat{\beta}<0\right\}$, shown in Fig. 3b. The idea is thus to perform an approximated FL only when the system trajectories come close to a singular point. Clearly, in this case, the chosen $\vartheta_{r}$ no longer guarantees satisfaction of (21). Nonetheless, under proper choice of $\omega^{\text {ref }}$, and deloading technique, approximation (23) may occur only during transients. We can summarize the results in this subsection by stating the following

Theorem 3. Given system (17), controlled via (20), and (22), where the $\beta$ function is replaced with (23). For any initial condition $\left(\omega_{r}(0), \vartheta(0)\right) \in \hat{\Lambda}$, the 


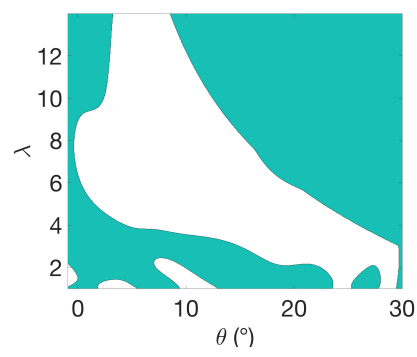

(a) $\Lambda$, white area, set of $(\lambda, \vartheta)$ such that $\beta(\lambda, \vartheta)<0$.

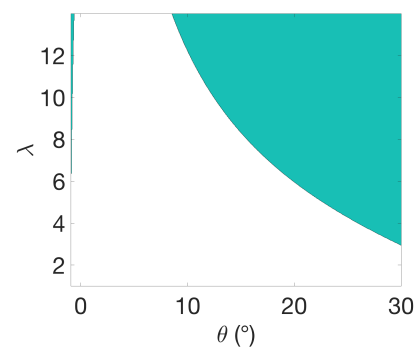

(b) $\hat{\Lambda}$, white area, set of $(\lambda, \vartheta)$ such that $\hat{\beta}(\lambda, \vartheta)<0$.

Fig. 3. Singular points with and without $\beta$ approximation.

system trajectories are bounded if parameters $b_{0}, \epsilon_{1}$, and $\varrho$ are chosen such that $\epsilon_{1}>0$ is sufficiently small, $\varrho>1$, and $b_{0}>-\frac{\varrho}{1-\varrho}$. In addition, if $\exists \bar{t} \geq 0$ such that $\frac{\partial C_{q}}{\partial \vartheta}(\lambda(t), \vartheta(t))<0 \forall t \geq \bar{t}$, then $\lim _{t \rightarrow \infty}\left|P^{r e f}(t)-P(t)\right|=0$.

Proof. First of all, initial conditions in $\hat{\Lambda}$ imply $\hat{\beta}(0)<0$, then $\epsilon_{1}>0, \varrho>1$ allow $\hat{\beta}(t)<0 \forall t \geq 0$. In particular $\hat{\beta}(t) \neq 0 \forall t \geq 0$, thus (22) is well-defined. Note that initial conditions considered in Problem 1 satisfy $\hat{\beta}(0)<0$, belonging to $\hat{\Lambda}$ shown in Fig. 3b. The system dynamics in closed loop is given by

$$
\dot{\varepsilon}_{p}=\left(-b_{0}+1-\frac{\beta}{\beta+\beta_{\varepsilon}}\right) \varepsilon_{p}+\left(1-\frac{\beta}{\beta+\beta_{\varepsilon}}\right) \varphi(\varsigma)
$$

where we named $\beta_{\varepsilon} \triangleq \hat{\beta}-\beta$, and $\varphi(\varsigma)$ the function composed of all the terms appearing in the right factor of (22) deprived of the term $b_{0} \varepsilon_{p}$, and being $\varsigma \triangleq\left(\omega_{r}, v, \vartheta_{s}, \dot{v}, w, \dot{w}, \dot{P}^{r e f}\right)$. The term $\left(1-\frac{\beta}{\beta+\beta_{\varepsilon}}\right) \varphi(\varsigma)$ is bounded in the trajectories thanks to the choice of $\beta_{\epsilon}$, and being $\varphi$ a continuous function on a compact set. The latter is compact because the wind is limited, $w, \dot{w}, \dot{P}^{r e f}$ are chosen to be so, $\omega_{r}$ is bounded thanks to (20), and term $\frac{d \sigma}{d \vartheta_{s}} \vartheta_{s}$ is bounded. Thus it will be considered as a bounded input of (24) to simplify the analysis. Finally system $(24)$ with $\varphi(\varsigma) \equiv 0$, given by

$$
\dot{\varepsilon}_{p}= \begin{cases}\left(-b_{0}+1-\frac{1}{\epsilon_{1}}\right) \varepsilon_{p} & \text { if } \beta=0 \\ \left(-b_{0}+1-\frac{1}{1-\varrho}\right) \varepsilon_{p} & \text { if } \beta>0 \\ -b_{0} \varepsilon_{p} & \text { otherwise }\end{cases}
$$

is stable if, for instance, we choose $b_{0}>-\frac{\varrho}{1-\varrho}$, and $\epsilon_{1}<1$. This can be proved by choosing $V\left(\varepsilon_{p}\right) \triangleq \frac{1}{2} \varepsilon_{p}^{2}$ as a common Lyapunov function for the family of 
systems (25), (see [29]). Eventually, if for some $\bar{t} \geq 0: \frac{\partial C_{q}}{\partial \vartheta}<0 \forall t \geq \bar{t}$, then (24) reduces to $\dot{\varepsilon}_{p}=-b_{0} \varepsilon_{p}$, thus $P \rightarrow P^{r e f}$ for $t \rightarrow \infty$.

Remark 4. Concerning $\omega^{r e f}$, we make the choice to use the MPPT signal $\omega_{r}^{o}=$ $\frac{\lambda^{o} v}{R}$ sufficiently filtered of its high frequency components. There are different motivations to support this choice. First of all, if $v$ varies rapidly so it does $\omega_{r}^{o}$, then if we consider $\omega^{r e f}=\omega_{r}^{o}$, its variation would directly effect $\vartheta_{r}$ via (22), and in turns $\vartheta$. This fact risks to make $\vartheta$ hit the saturation constraints of the sigmoid function, and more in general, to not let the constraints on $\dot{\vartheta}$ be respected, as in this framework they are only verified a posteriori. Secondly, if $\omega^{r e f}$ varies too rapidly, by empirical results it turns out that the closed-loop system trajectories are more likely to approach singular points, letting the activation of $\varepsilon(\lambda, \vartheta)$ defined in (23), and not allowing satisfaction of (21). On the other hand, filtering $\omega_{r}^{o}$ let $(22)$ be defined, i.e. it fulfils the requirement of tracking the desired deloaded power reference. The physical explanation of this fact is that for a given deloaded $P^{r e f}$ there exist infinite pairs $\left(\omega_{r}, \vartheta\right) \in \mathcal{X}$ that let a WT track it, (see e.g. $[30,31])$, and by filtering $\omega_{r}^{o}$, we are simply considering another possible choice of couples $\left(\omega_{r}, \vartheta\right)$ than the one in which $\omega_{r}=\omega_{r}^{o}$.

\subsection{Disturbance Effect and Additional Local Control Settings}

Assumption 4 Trajectories of the closed-loop system described by (17), (20), (22) verify $\frac{\partial C_{q}}{\partial \vartheta}<0$.

As previously mentioned, we assume that turbine $i$ local controller is able to measure $v_{m, i}$ such that $v_{i}=v_{m, i}+v_{d, i}$. The effect of $v_{d, i}$ on the closed loop dynamics can be thus approximated as

$$
\dot{\varepsilon}_{p, i}=-b_{0} \varepsilon_{p, i}+\mu_{1}\left(\hat{\zeta}_{i}\right) v_{d, i}+\mu_{2}\left(\hat{\zeta}_{i}\right) v_{d, i}^{2}+\mu_{3}\left(\hat{\zeta}_{i}\right) \dot{v}_{d, i}
$$

obtained via first order Taylor expansion of the functions depending on $v_{i}$, in a neighborhood of $v_{m, i}$, e.g. $T_{r}\left(v_{i}\right) \cong T_{r}\left(v_{m, i}\right)+\frac{\partial T_{r}}{\partial v}\left(v_{m, i}\right) v_{d, i}$, and where $\hat{\zeta}_{i} \triangleq$ $\left(\omega_{r, i}, \vartheta_{i}, v_{m, i}, \dot{v}_{m, i}\right)$. Functions $\mu_{1}, \mu_{2}, \mu_{3}$ are not reported here for the sake of brevity. According to Assumption 3 we neglect $\mu_{3}\left(\hat{\zeta}_{i}\right) \dot{v}_{d, i}$. Moreover, by numerical simulation, the contribution of term $\mu_{2} v_{d, i}^{2}$ can be neglected with respect to $\mu_{1} v_{d, i}$. On the compact set on which $\mu_{1}$ is defined, the function satisfies $\mu_{1, \min } \leq$ $\mu_{1} \leq 0$, thus in the sequel we treat $\mu_{1}$ as a parametric uncertainty, and we drop its dependency on $\hat{\zeta}_{i}$ for ease of notation. Being interested in a discrete-time communication set-up among the WTs we shall consider system (26) discretized at sampling time $T_{s}$, given by

$$
\begin{aligned}
{\left[\begin{array}{c}
\xi_{i}(k+1) \\
P_{i}(k+1)
\end{array}\right]=} & {\left[\begin{array}{cc}
0 & 0 \\
-1 & \left(1-T_{s} b_{0}\right)
\end{array}\right]\left[\begin{array}{l}
\xi_{i}(k) \\
P_{i}(k)
\end{array}\right]+} \\
& {\left[\begin{array}{c}
1 \\
\left(1+T_{s} b_{0}\right)
\end{array}\right] P_{i}^{r e f}(k)+\left[\begin{array}{c}
0 \\
\mu_{1} T_{s}
\end{array}\right] v_{d, i}(k) }
\end{aligned}
$$


where we used Euler approximation, $\dot{P}_{i}^{r e f} \cong \frac{\left(P_{i}^{r e f}(k)-P_{i}^{r e f}(k-1)\right)}{T_{s}}$, and we named $\xi_{i}(k) \triangleq P_{i}^{r e f}(k-1)$. System $(27)$ is required to track the optimal power reference provided by the higher optimization step according to (18). For the moment, let us assume that $P_{i}^{f w}, i=1, \ldots, N$ are not affected by wind measurement error, i.e. $P_{i}^{f w}\left(v_{i}\right)$. Because of the presence of $v_{d, i}$ in (27), affecting the controlled WT dynamics, simply setting $P_{i}^{r e f}=P_{i}^{f w}$ does not guarantee $P_{i}$ to asymptotically converge to $P_{i}^{f w}$, nor the satisfaction of relationship (19), describing the optimal relative power sharing values among the WTs. Under the assumption of communicating WTs on an undirected connected graph $\mathcal{G}$, whose associated Laplacian matrix is $\mathcal{L}$, one could think to exploit the leaderless PIDlike distributed protocol developed in Section 2 to reduce the effect of $v_{d}$ on the weighted consensus among the WTs, by acting on $P_{i}^{\text {ref }}$. Nonetheless, as pointed out in Remark 2, still the consensus function to which the system converges depends in the disturbance signals. As a result, even if relative distances on power values according to (19) are respected thanks to consensus control, there is no general guarantee for the power values to reach $P_{i}^{f w}$. This is why before continuing our analysis on WF consensus control, we introduce an additional local PI loop to system (27) to let convergence to the absolute power value $P_{i}^{f w}$. Note that the two integral actions, namely the internal loop one controlling $P_{i}$ to $P_{i}^{f w}$, and the distributed control one controlling $P_{i}$ to satisfy condition (19), are not in contradiction if $P_{i}^{f w}$ are measurement error free. Indeed, by naming $P^{f w} \triangleq \operatorname{col}\left(P_{1}^{f w}, \ldots, P_{N}^{f w}\right)$, then by construction, $\mathcal{L} D P^{f w}=\mathbf{0}$, where recall $D \triangleq \operatorname{diag}\left(\frac{1}{\chi_{1}}, \cdots, \frac{1}{\chi_{N}}\right)$, which is exactly the weighted consensus condition seek by the distributed protocol. As a consequence, in such case of perfect information on $P_{i}^{f w}$, the distributed consensus is not necessary to let condition (19) be satisfied, as it is already ensured by the internal PI. However, even in this case, consensus control can be employed to enhance closed-loop performance.

The above discussion holds for the case in which the wind disturbance $v_{d, i}$ only affects the system equations as shown in (27). However, $v_{d, i}$ has also a role in the computation of $P_{i}^{f w}$, since in reality we have $P_{i}^{f w}\left(v_{m, i}\right)$. In this case, condition $\mathcal{L} D P^{f w}=\mathbf{0}$ generally does not hold, i.e. the power references provided to the local WT controller may not let satisfaction of the optimal relative power sharing. Under these circumstances, the distributed PID does have a role in forcing weighted consensus among the WTs. Moreover, the two integral actions may come to a conflict. In the following we decide to give priority to consensus seeking by allowing the distributed control action modify the local error $P_{i}^{f w}-P_{i}$. This is achieved by considering the dashed arrow shown in the control scheme of Fig. 4, where the overall WT control is illustrated. The idea behind this choice is that zero-mean disturbances on the local power references $P_{i}^{f w}$ can be globally evened out by enforcing relative power distances among the network of wind turbines.

By naming $K_{I}^{l}$, and $K_{P}^{l}$ respectively the integral, and proportional gains of the PI, we can write $(27)$ in closed-loop as $x_{i}(k+1)=A x_{i}(k)+B_{2} u_{i}(k)+$ 


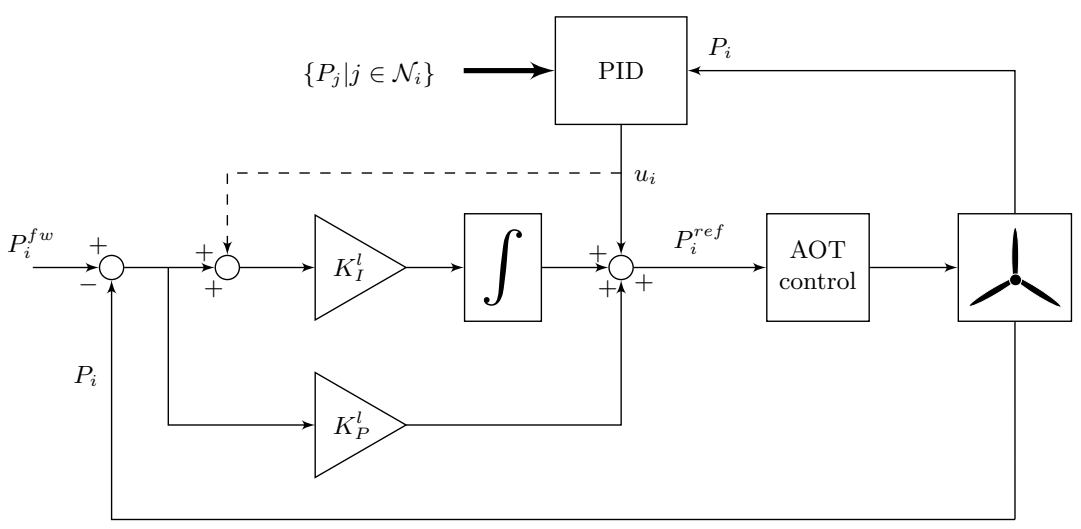

Fig. 4. WT control scheme: the local control is composed of an AOT step and of a PI; the distributed control has a PID structure. Each WT $i$ receives $P$ values from its neighbors, i.e. WTs $j \in \mathcal{N}_{i}$.

$B_{f w} P_{i}^{f w}(k)+B_{w} v_{d, i}(k)$, where

$$
\begin{aligned}
& A \triangleq\left[\begin{array}{ccc}
1 & 0 & -K_{I}^{l} T_{s} \\
1 & 0 & -K_{P}^{l} \\
\left(1+T_{s} b_{0}\right) & -1 & -T_{s} b_{0}
\end{array}\right], B_{w} \triangleq\left[\begin{array}{c}
0 \\
0 \\
\mu_{1} T_{s}
\end{array}\right] \\
& B_{2} \triangleq\left[\begin{array}{c}
K_{I}^{l} T_{s} \\
1 \\
\left(1+T_{s} b_{0}\right)
\end{array}\right], B_{f w} \triangleq\left[\begin{array}{c}
K_{I}^{l} T_{s} \\
K_{P}^{l} \\
\left(1+T_{s} b_{0}\right) K_{P}^{l}
\end{array}\right]
\end{aligned}
$$

and where we named $x_{i} \triangleq \operatorname{col}\left(\delta_{i}, \xi_{i}, P_{i}\right)$, being $\delta_{i}$ the state of the local integral action, $P_{i}^{f w}$ a forward signal, and $u_{i}$ is left as a degree of freedom to let distributed control. Note that other choices for the introduction of $u_{i}$ in the internal WT control loop would have been possible.

\subsection{Wind Farm Distributed Protocols}

Leaderless Consensus As previously mentioned, consensus control over the WF can be employed to let satisfaction of relationship (19) over a set of $N$ controlled WTs of the form of (28). This can be done by making use of the tools concerning leaderless consensus shown in Section 2. This is simply obtained by choosing as $A, B_{2}$ the matrices of (28), $C=\left[\begin{array}{lll}0 & 0 & 1\end{array}\right]$, i.e. $P_{i}$ is the measured and controlled output, and $B_{1}=\left[\begin{array}{ll}B_{f w} & B_{w}\end{array}\right]$, i.e. $P_{i}^{f w}$, and $v_{d, i}$ are both considered as disturbances with respect to the weighted consensus.

Remark 5. The considered control approach relies on Assumption 3. In particular disturbances $v_{d, i}$ are supposed to be zero-mean signals. If there exist WTs for which $v_{d, i}$ has not zero mean, then the corresponding absolute power reference 


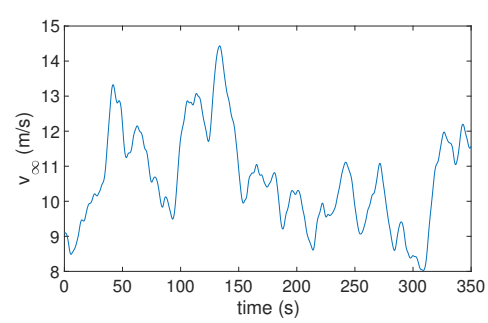

(a) Free stream wind speed.

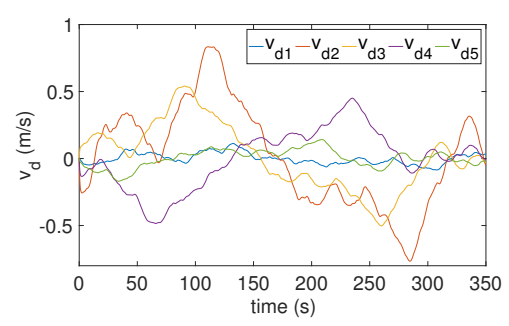

(b) Wind disturbances speed.

Fig. 5. Wind signals.

$P_{i}^{f w}$ would be in average different from the optimal one. Unfortunately under these circumstances, there is no hope for the optimal absolute power sharing to be satisfied by the only means of consensus control, even if optimal relative power sharing condition (19) is satisfied. This is due to the fact that in a network of agents communicating on an undirected graph, each of them has a role in determining the consensus function to which they converge. Thus, WTs affected by nonzero-mean wind disturbance would make the whole network deviate from the optimal absolute power values in average.

Leader-follower Consensus Since leaderless consensus technique fails to restore optimal absolute power references when some WTs in the wind farm are subject to nonzero-mean wind measurement errors, if the WTs being affected by nonzero-mean wind disturbances can be detected and isolated then, for instance, the leader-follower techniques developed in Section 3 can be employed to restore the optimal power references in the concerned WTs. This could be achieved by letting the faulty WTs be follower agents, and the unfaulty WT network serve as a leader.

If, for the sake of simplicity, we consider only one WT not to be affected by absolute power reference error, and we thus let it be the only leader in the WF, then Theorem 2 can be applied by choosing $A, B_{2}$ the matrices of (28), $C=\left[\begin{array}{lll}0 & 0 & 1\end{array}\right]$, and $B_{1}=B_{f w}$. In addition to what shown in the leader-follower development of Section 3, in this case either the followers and the leader are additionally subject to disturbances via matrix $\left[\begin{array}{ll}B_{f w} & B_{w}\end{array}\right]$, i.e. $P_{i}^{f w}$, and $v_{d, i}$ are both considered as disturbances with respect to the weighted consensus.

\section{Simulation}

In the following we propose some numerical simulations to show the overall WF control performance in both leaderless and leader-follower modes. In the considered simulation test, the system is subject to two sources of model-plant mismatches. The first one is caused by differences between the polynomial $C_{p}$ approximation used for the AOT-based controller design and the CART power 


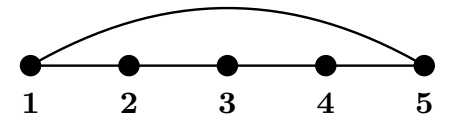

(a) Leaderless consensus graph.

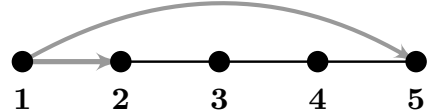

(b) Leader-follower consensus graph.

Fig. 6. WF example communication graph.

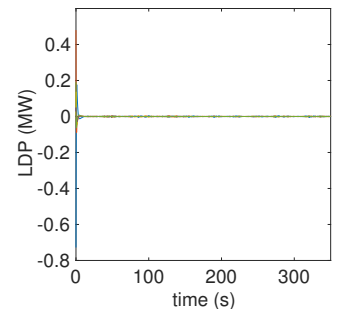

(a) $\mathcal{L} D P$ signals. They are expected to reach the origin.

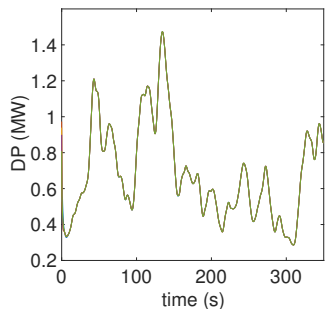

(b) $D P$ signals. They are expected to reach a common value.

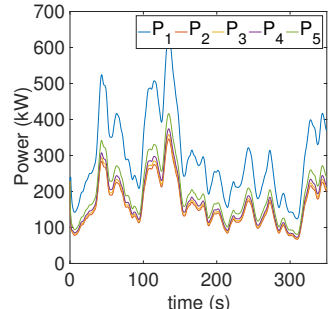

(c) WT powers. They are expected to keep constant relative distances.

Fig. 7. Wind farm leaderless control.

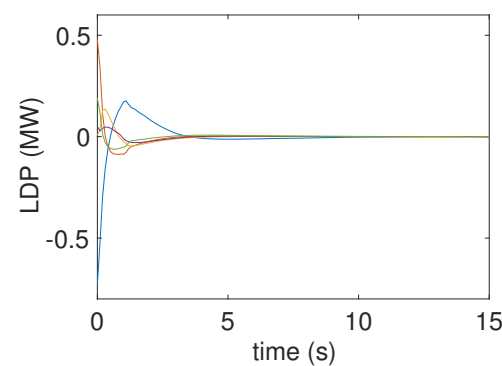

(a) $\mathcal{L} D P$ signals. They are expected to reach the origin.

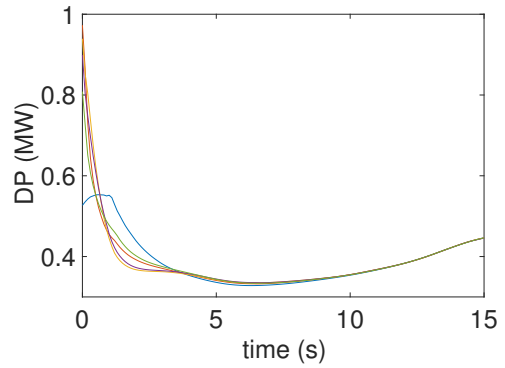

(b) $D P$ signals. They are expected to reach a common value.

Fig. 8. Zoom on $\mathcal{L} D P$ and $D P$ during WF leaderless control.

coefficient given as a lookup table, and shown in Fig. 2a. The second one is given by the way $v_{d, i}$ acts on WT $i$. Indeed, each $v_{d, i}$ affects the according WT $i$ dynamics via the mechanical power $P_{r}$. This causes an additional mismatch as recall that $v_{d, i}$ effect was approximated in Subsection 5.2, leading to the approximated model of system (27). We consider a WF composed of $N=6$ WTs aligned one after the other according to the wind direction. We suppose the 6-th WT to be the last one of the row according to the wind direction. Thus 


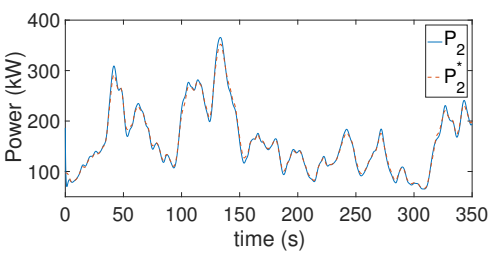

(a) WT 2 restored power.

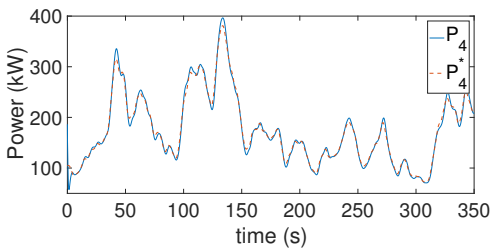

(c) WT 4 restored power.

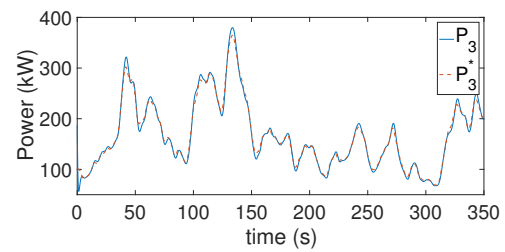

(b) WT 3 restored power.

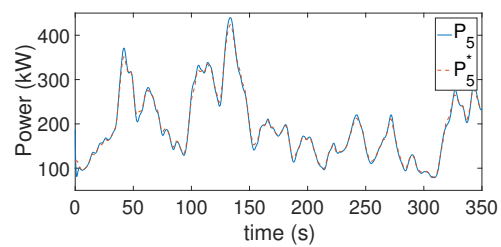

(d) WT 5 restored power.

Fig. 9. Wind farm leader-follower control.

it is required to always operate in classic MPPT mode. In the following tests, it is supposed to not intervene in the consensus control, and its power signals will not be reported. Concerning leaderless control, the remaining WTs are supposed to communicate on the undirected graph shown in Fig. 6a. The free stream wind speed $v_{\infty}$ blowing in front of WT 1 is chosen to have the profile of Fig. 5a. The other wind speed signals $v_{i}, i=2, \ldots, 5$ are obtained from $v_{\infty}$ according to the wake model, (e.g. see [19]). The local WT PI gains are set equal to $K_{P}^{l}=0.2$, and $K_{I}^{l}=0.8$, while the PID gains for the aforementioned multi-agent system found via Theorem 1 are $K_{p}=0.0072, K_{i}=-0.0638, K_{d}=0.0013$, and they allow weighted consensus achievement with performance index $\tau=24.5$. The disturbance $v_{d} \triangleq \operatorname{col}(d, 1, \ldots, d, 5)$ affecting the system is chosen to be the one shown in Fig. 5b. In this case $P^{f w}$ is computed on the available measurements $v_{m, i}$. Thus, they are corrupted by measurement error, and they do not respect the optimal relative power sharing condition (19). For this simulation we show signals $\mathcal{L} D P, D P$, and $P$, where $P \triangleq \operatorname{col}\left(P_{1}, \ldots, P_{5}\right)$. Indeed consensus is reached when $\mathcal{L} D P=\mathbf{0}$, which can be alternatively seen as weighted power signals $D P$ reaching a common value, or as power signals $P$ reaching defined constant relative distances. These are illustrated in Fig. 7, from which we can also notice that the aforesaid model-plant mismatches, as well as temporary dissatisfaction of Assumption 4, cause persistent small oscillations around the reached weighted consensus. For the sake of clarity, $\mathcal{L} D P$, and $D P$ are also shown in a zoomed window in Fig. 8.

Eventually, we aim at showing how optimal absolute power references $P_{i}^{\star}$ can be restored via leader-follower consensus when some WTs in the WF are affected by power reference error. In order to do, we consider a simple example in which the only first WT in the row is error-free, and thus acts as the leader. In such 
case, we consider the WF communication graph to be modified according to Fig. 6b, where the leader, WT 1, can communicate with WT 2 and WT 5 directly. The remaining WTs from 2 to 5 are followers and they can communicate on an undirected connected graph. We suppose the leader $v_{d, 1}$ to be equal to the one shown in Fig. 5b, whereas the followers wind disturbances are nonzeromean signals of the form of $v_{d, i}=\bar{v}_{d, i}+\tilde{v}_{d, i}$, where $\tilde{v}_{d, i}$ is a zero-mean signal, and $\bar{v}_{d, i}$ a constant nonzero value. $\tilde{v}_{d, i}, i=2, \ldots, 5$ are supposed to be equal to the corresponding $i$-th signal in Fig. $5 \mathrm{~b}$ used in the previous simulation, while $\bar{v}_{d, i}$ are such that $P_{2}^{f w}=80 \% P_{2}^{\star}, P_{3}^{f w}=60 \% P_{3}^{\star}, P_{4}^{f w}=30 \% P_{4}^{\star}, P_{5}^{f w}=$ $40 \% P_{5}^{\star}$, i.e. without leader-follower control they would track a not optimal power reference. By employing fast leader-follower weighted consensus technique, the PID gains are $K_{p}=0.0082, K_{i}=-0.0656$, and $K_{d}=-0.0028$, and they allow a performance index equal to $\tau=24.5$. Simulation results are illustrated in Fig. 9, where the red dashed signals represent the original optimal power reference $P_{i}^{\star}$, and the blue solid line the obtained power output via consensus control. We are able to conclude that consensus control shows good performance in restoring the optimal absolute power values. Small persistent errors are due to modelplant mismatches, by the fact that the PID structure cannot reject general timevarying reference state, and because wind disturbance $\tilde{v}_{d, i}$ also affect the system consensus function.

\section{Conclusion}

We presented a PID-like distributed protocol for general LTI MIMO discretetime agents. By employing LMIs we showed how the controller gains can be tuned to solve two different, yet similar, problems, namely a leaderless under system disturbances and a leader-follower under time-varying reference state weighted consensus problem. The distributed control approaches were then applied to treat some issues concerning the wind farm power maximization problem under wake effect. In order to do so, first an approximated AOT control technique allows a WT to track a general deloaded power reference by acting on both the rotor speed and the pitch angle. Leaderless control technique was then used to average out zero-mean wind disturbances from the optimal WF power sharing, while we showed how leader-follower control can be applied to restore the optimal power sharing in the case of power reference errors.

In the near future it would be interesting to consider other LMI approaches, such as $\mathcal{H}_{\infty}$ loop-shaping, to tune a distributed controller that could take into account prior knowledge about system disturbances for a better rejection on consensus reaching. Concerning the wind farm control problem, consensus control represents a fairly new approach, and ti may lead to a great variety of other applications, such as the distributed estimation of the wind filed within a wind farm.

Acknowledgment This study has been carried out in the RISEGrid Institute (www.supelec.fr/342p38091/risegrid-en.html), joint program between CentraleSupélec and EDF ('Electricité de France') on smarter electric grids. 


\section{Appendix}

\section{Appendix 1}

A directed graph $\mathcal{G}$, called digraph, is a pair $(\mathcal{V}, \mathcal{E})$, where $\mathcal{V}=\{1, \ldots, N\}$ is the set of nodes, and $\mathcal{E} \subseteq \mathcal{V} \times \mathcal{V}$ is the set of unordered pairs of nodes, named edges. Two nodes $i, j$ are said to be adjacent if $(i, j) \in \mathcal{E}$. In such case the communication is supposed to be directed from $i$ to $j$. We additionally define $\mathcal{N}_{i}$ to be the set of neighbors of node $i$, i.e. $\mathcal{N}_{i} \triangleq\{j \in \mathcal{V}:(j, i) \in \mathcal{E}\}$. The weighted adjacency matrix $\mathcal{A}=\left[a_{i j}\right] \in \mathbb{R}^{N \times N}$ associated with the digraph $\mathcal{G}$, is defined by $a_{i i}=0$, i.e. self-loops are not allowed, and $a_{i j}>0$ if $(i, j) \in \mathcal{E}$. The Laplacian matrix $\mathcal{L}=\left[l_{i j}\right] \in \mathbb{R}^{N \times N}$ is defined as $l_{i i}=\sum_{j \neq i} a_{i j}$ and $l_{i j}=-a_{i j}, i \neq j$. Typically, if the adjacency matrix is not weighted, then we simply assign $a_{i j}=1$ if $(i, j) \in \mathcal{E}$. Moreover, under the assumption of undirected graph, $(i, j) \in \mathcal{E}$ implies that $(j, i) \in \mathcal{E}$ too. In this report an undirected graph is always considered to be not weighted. An undirected graph is connected if there exists a path between every pair of distinct nodes, otherwise it is disconnected. If there exist an edge between any two nodes, the graph is said to be complete. We provide the following useful lemmas.

Lemma 1. [32] The Laplacian matrix has the following properties: (i) if $\mathcal{A}$ refers to an undirected graph, then $\mathcal{L}$ is symmetric and all its eigenvalues are either strictly positive or equal to 0 , and 1 is the corresponding eigenvector to 0 ;

(ii) 0 is a simple eigenvalue of $\mathcal{L}$ if and only if the graph is connected.

Lemma 2. [33] Let $\overline{\mathcal{L}}=\left[\bar{l}_{i j}\right] \in \mathbb{R}^{N \times N}$ be a Laplacian matrix such that $\bar{l}_{i j}=$ $N-1 / N$ if $i=j$, and $\bar{l}_{i j}=-1 / N$ otherwise, then the following hold: $(i)$ the eigenvalues of $\overline{\mathcal{L}}$ are 1 with multiplicity $N-1$, and 0 with multiplicity $1 . \mathbf{1}^{\top}$ and $\mathbf{1}$ are respectively the left and right eigenvector associated to eigenvalue 0 ; (ii) there exists an orthogonal matrix $U \in \mathbb{R}^{N \times N}$, i.e. $U: U^{\top} U=U U^{\top}=I$, and whose last column is equal to $1 / \sqrt{N}$, such that for any Laplacian matrix $\mathcal{L}$ associated to any undirected graph we have

$$
\begin{aligned}
U^{\top} \overline{\mathcal{L}} U & =\left[\begin{array}{cc}
I_{N-1} & 0_{(N-1) \times 1} \\
0_{1 \times(N-1)} & 0
\end{array}\right] \triangleq \bar{\Lambda}, \\
U^{\top} \mathcal{L} U & =\left[\begin{array}{cc}
\mathcal{L}_{1} & 0_{(N-1) \times 1} \\
0_{1 \times(N-1)} & 0
\end{array}\right]
\end{aligned}
$$

where $\mathcal{L}_{1} \in \mathbb{R}^{(N-1) \times(N-1)}$ is symmetric and positive definite if the graph is connected.

Moreover we deduce the following extension of Lemma 2.

Lemma 3. Let $\mathcal{L} \in \mathbb{R}^{N \times N}$ be the Laplacian matrix associated to an undirected connected graph, and let $D \in \mathbb{R}^{N \times N} \succ 0$, and symmetric, then the following hold: $(i) \hat{\mathcal{L}} \triangleq D \mathcal{L} \succeq 0$, all its eigenvalues are real, and 0 is a simple eigenvalue 
with associated eigenvector 1; (ii) consider the orthogonal matrix $U \in \mathbb{R}^{N \times N}$ defined in Lemma 2, then

$$
U^{\top} \hat{\mathcal{L}} U=\left[\begin{array}{cc}
\hat{\mathcal{L}}_{1} & 0_{(N-1) \times 1} \\
* & 0
\end{array}\right]
$$

where $*$ indicates a generally nonempty row, $\hat{\mathcal{L}}_{1} \in \mathbb{R}^{(N-1) \times(N-1)} \succ 0$, and its eigenvalues are real.

Proof. We have that $D \mathcal{L}=D^{1 / 2}\left(D^{1 / 2} \mathcal{L} D^{1 / 2}\right) D^{-1 / 2}$, thus $D \mathcal{L}$ is similar to a symmetric semi-definite positive matrix, so its eigenvalues are positive real. $\hat{\mathcal{L}}$ preserves the 0 eigenvalue, and its associated eigenvector $\mathbf{1}$, as $D \mathcal{L} \mathbf{1}=\mathbf{0}$. 0 is a simple eigenvalue as $D$ is nonsingular, and $\mathcal{L}$ has one simple 0 eigenvalue by hypothesis. The last column of $U^{\top} \hat{\mathcal{L}} U$ has all its entries equal to 0 because the last column of $U$ is $1 / \sqrt{N}$. Being $U^{\top} \hat{\mathcal{L}} U$ block triangular, and similar to $\hat{\mathcal{L}}, \hat{\mathcal{L}}_{1}$ has all real strictly positive eigenvalues.

Lemma 4. Given two symmetric matrices $A$, and $B$ of equal dimension such that $A \succeq 0$, and $B \succ 0$; then $\sigma(A B) \subset \mathbb{C}_{\geq 0}$.

Proof. It exists the symmetric matrix $B^{1 / 2}: B^{1 / 2} B^{1 / 2}=B$, where $B^{1 / 2} \succ 0$, and it exists $B^{-1 / 2}: B^{-1 / 2} B^{1 / 2}=B^{1 / 2} B^{-1 / 2}=I$. We have that $A B=A B^{1 / 2} B^{1 / 2}=$ $B^{-1 / 2}\left(B^{1 / 2} A B^{1 / 2}\right) B^{1 / 2}$, thus $\sigma(A B) \equiv \sigma\left(B^{1 / 2} A B^{1 / 2}\right)$. Moreover $B^{1 / 2} A B^{1 / 2} \succeq 0$ because $A \succeq 0$ and $B^{1 / 2}$ is symmetric. Thus $\sigma\left(B^{1 / 2} A B^{1 / 2}\right) \subset \mathbb{C}_{\geq 0}$.

Lemma 5. Given two symmetric matrices $A$, and $B$ of equal dimension; if $A \succ$ 0 then $A B$ is diagonalizable in $\mathbb{R}$.

Proof. It exists the symmetric matrix $A^{1 / 2}: A^{1 / 2} A^{1 / 2}=A$, where $A^{1 / 2} \succ 0$, and it exists $A^{-1 / 2}: A^{-1 / 2} A^{1 / 2}=A^{1 / 2} A^{-1 / 2}=I$. We have $A^{-1 / 2} A B A^{1 / 2}=A^{1 / 2} B A^{1 / 2}$, and the latter is symmetric. Thus $A B$ is similar to a symmetric matrix, so it is diagonalizable in $\mathbb{R}$.

We recall the following lemma on Kronecker product $\otimes$

Lemma 6. [34] Suppose that $U \in \mathbb{R}^{p \times p}, V \in \mathbb{R}^{q \times q}, X \in \mathbb{R}^{p \times p}$, and $Y \in \mathbb{R}^{q \times q}$. The following hold: $(i)(U \otimes V)(X \otimes Y)=U X \otimes V Y$; (ii) suppose $U$, and $V$ invertible, then $(U \otimes V)^{-1}=U^{-1} \otimes V^{-1}$.

\section{Appendix 2}

In the following we report the cited theorem of [24]. Consider the system of equations

$$
\left\{\begin{array}{l}
x^{+}=A x+B u \\
y=C x
\end{array}\right.
$$

where $A \in \mathbb{R}^{n \times n}, B \in \mathbb{R}^{n \times l}, C \in \mathbb{R}^{m \times n}, x \triangleq x(k) \in \mathbb{R}^{n}$ and $x^{+} \triangleq x(k+1) \in \mathbb{R}^{n}$ are respectively the system state at the current step $k$, and at the next step $k+1$, 
$u \triangleq u(k) \in \mathbb{R}^{l}$ is the control input, and $y \triangleq y(k) \in \mathbb{R}^{m}$ is the measured and controlled output. Define the matrices $C_{c l} \triangleq\left[\begin{array}{ll}C & 0_{r \times(2 l)}\end{array}\right], K \triangleq\left[\begin{array}{ll}D_{c}^{\top} & B_{c}^{\top}\end{array}\right]^{\top}$, and

$$
\tilde{A} \triangleq\left[\begin{array}{cc}
A & B C_{c} \\
0_{2 l \times n} & A_{c}
\end{array}\right]
$$

where $A_{c}, B_{c}, C_{c}$, and $D_{c}$ are defined in (3). Assuming $B$ to be of full column rank without loss of generality, there exists an invertible $T_{b} \in \mathbb{R}^{n \times n}: T_{b} B=$ $\left[\begin{array}{ll}0_{l \times(n-l)} & I_{l \times l}\end{array}\right]^{\top}$. Finally define

$$
T \triangleq\left[\begin{array}{cc}
T_{b} & 0_{n \times 2 l} \\
0_{2 l \times n} & I_{2 l \times 2 l}
\end{array}\right]
$$

Thus, we have the following theorem

Theorem 4. Consider system (29). If there exists a positive definite matrix $P \in \mathbb{R}^{\bar{n} \times \bar{n}}$, and a matrix

$$
J=\left[\begin{array}{cc}
J_{11} & 0_{(\bar{n}-q) \times 3 l} \\
J_{21} & J_{22}
\end{array}\right]
$$

$J_{22} \in \mathbb{R}^{3 l \times 3 l}$, and $X \in \mathbb{R}^{3 l \times m}$, and we further name

$$
\Omega \triangleq\left[\begin{array}{cc}
0_{(\bar{n}-3 l) \times n} & 0_{(\bar{n}-3 l) \times 2 l} \\
X C & 0_{3 l \times 2 l}
\end{array}\right]
$$

such that the following LMI has a solution

$$
\left[\begin{array}{c}
b P \\
\Omega+J T \tilde{A}+a J T \\
\Omega\left(J T+(J T)^{\top}-P\right)
\end{array}\right]>0
$$

where * indicates the transposed of the opposite term with respect to the matrix diagonal, and if $J$ is nonsingular, then by choosing $K=J_{22}^{-1} X$, the eigenvalues of the following matrix

$$
A_{c l} \triangleq\left[\begin{array}{cc}
\left(A+B D_{c} C\right) & B_{2} C_{c} \\
B_{c} C & A_{c}
\end{array}\right]
$$

lie in the region $\mathcal{F}_{D} \triangleq\left\{(\Re[\lambda], \Im[\lambda]):(\Re[\lambda]+a)^{2}+\Im[\lambda]^{2}<b^{2}\right\}$.

\section{References}

1. Ren, W., Beard, R.W.: Distributed consensus in multi-vehicle cooperative control. Springer (2008)

2. Xi, J., Cai, N., Zhong, Y.: Consensus problems for high-order linear time-invariant swarm systems. Physica A: Statistical Mechanics and its Applications 389(24), 5619-5627 (2010)

3. Li, Z., Ren, W., Liu, X., Fu, M.: Distributed containment control of multi-agent systems with general linear dynamics in the presence of multiple leaders. International Journal of Robust and Nonlinear Control 23(5), 534-547 (2013) 
4. Yang-Zhou, C., Yan-Rong, G., ZHANG, Y.X.: Partial stability approach to consensus problem of linear multi-agent systems. Acta Automatica Sinica 40(11), 2573$2584(2014)$

5. You, K., Xie, L.: Network topology and communication data rate for consensusability of discrete-time multi-agent systems. IEEE Transactions on Automatic Control 56(10), 2262-2275 (2011)

6. Su, Y., Huang, J.: Two consensus problems for discrete-time multi-agent systems with switching network topology. Automatica 48(9), 1988-1997 (2012)

7. Ge, Y., Chen, Y., Zhang, Y., He, Z.: State consensus analysis and design for highorder discrete-time linear multiagent systems. Mathematical Problems in Engineering 2013 (2013)

8. Li, Z., Duan, Z., Xie, L., Liu, X.: Distributed robust control of linear multiagent systems with parameter uncertainties. International Journal of Control 85(8), 1039-1050 (2012)

9. Oh, K.K., Moore, K.L., Ahn, H.S.: Disturbance attenuation in a consensus network of identical linear systems: An approach. IEEE Transactions on Automatic Control 59(8), 2164-2169 (2014)

10. Li, Z., Duan, Z., Chen, G.: On $h_{\infty}$ and $h_{2}$ performance regions of multi-agent systems. Automatica 47(4), 797-803 (2011)

11. Wang, L., Gao, L.: $\mathrm{H}_{\infty}$ consensus control for discrete-time multi-agent systems with switching topology. Procedia Engineering 15, 601 - 607 (2011)

12. Xi, J., Shi, Z., Zhong, Y.: Output consensus analysis and design for high-order linear swarm systems: partial stability method. Automatica 48(9), 2335-2343 (2012)

13. Liu, Y., Jia, Y., Du, J., Yuan, S.: Dynamic output feedback control for consensus of multi-agent systems: an $\mathrm{h}_{\infty}$ approach. In: 2009 American Control Conference. pp. 4470-4475. IEEE (2009)

14. Carli, R., Chiuso, A., Schenato, L., Zampieri, S.: A pi consensus controller for networked clocks synchronization. IFAC Proceedings Volumes 41(2), 10289-10294 (2008)

15. Ou, L.L., Chen, J.J., Zhang, D.M., Zhang, L., Zhang, W.D.: Distributed $\mathrm{h}_{\infty}$ pid feedback for improving consensus performance of arbitrary-delayed multi-agent system. International Journal of Automation and Computing 11(2), 189-196 (2014)

16. Ren, W.: Multi-vehicle consensus with a time-varying reference state. Systems \& Control Letters 56(7), 474-483 (2007)

17. Cao, Y., Ren, W., Li, Y.: Distributed discrete-time coordinated tracking with a time-varying reference state and limited communication. Automatica 45(5), 12991305 (2009)

18. Gionfra, N., Sandou, G., Siguerdidjane, H., Faille, D.: A distributed pid-like consensus control for discrete-time multi-agent systems. In: ICINCO, 14th International Conference on Informatics in Control, Automation and Robotics (2017)

19. Park, J., Law, K.H.: Cooperative wind turbine control for maximizing wind farm power using sequential convex programming. Energy Conversion and Management 101, 295-316 (2015)

20. Zhang, W., Xu, Y., Liu, W., Ferrese, F., Liu, L.: Fully distributed coordination of multiple dfigs in a microgrid for load sharing. IEEE Transactions on Smart Grid $4(2), 806-815$ (2013)

21. Biegel, B., Madjidian, D., Spudic, V., Rantzer, A., Stoustrup, J.: Distributed lowcomplexity controller for wind power plant in derated operation. In: Control Applications (CCA), 2013 IEEE International Conference on. pp. 146-151. IEEE (2013)

22. Baros, S., Ilic, M.D.: Distributed torque control of deloaded wind dfigs for wind farm power output regulation. IEEE Transactions on Power Systems (99) (2017) 
23. Gionfra, N., Sandou, G., Siguerdidjane, H., Faille, D., Philippe, L.: A distributed consensus control under disturbances for wind farm power maximization. In: Decision and Control (CDC), 2017 IEEE 56th Annual Conference on. IEEE (2017)

24. Wu, Z., Iqbal, A., Amara, F.B.: Lmi-based multivariable pid controller design and its application to the control of the surface shape of magnetic fluid deformable mirrors. IEEE Transactions on Control Systems Technology 19(4), 717-729 (2011)

25. Boukhezzar, B., Siguerdidjane, H.: Nonlinear control of variable speed wind turbines for power regulation. In: Control Applications, 2005. CCA 2005. Proceedings of 2005 IEEE Conference on. pp. 114-119. IEEE (2005)

26. Gebraad, P.M., van Dam, F.C., van Wingerden, J.W.: A model-free distributed approach for wind plant control. In: American Control Conference (ACC), 2013. pp. 628-633. IEEE (2013)

27. Gionfra, N., Siguerdidjane, H., Sandou, G., Faille, D.: Hierarchical control of a wind farm for wake interaction minimization. IFAC-PapersOnLine 49(27), 330335 (2016)

28. Gionfra, N., Siguerdidjane, H., Sandou, G., Faille, D., Loevenbruck, P.: Combined feedback linearization and $\mathrm{mpc}$ for wind turbine power tracking. In: Control Applications (CCA), 2016 IEEE Conference on. pp. 52-57. IEEE (2016)

29. Liberzon, D.: Switching in systems and control. Springer Science \& Business Media (2012)

30. Yingcheng, X., Nengling, T.: Review of contribution to frequency control through variable speed wind turbine. Renewable Energy 36(6), 1671-1677 (2011)

31. Žertek, A., Verbič, G., Pantoš, M.: Optimised control approach for frequencycontrol contribution of variable speed wind turbines. IET Renewable power generation 6(1), 17-23 (2012)

32. Ren, W., Beard, R.W., et al.: Consensus seeking in multiagent systems under dynamically changing interaction topologies. IEEE Transactions on automatic control 50(5), 655-661 (2005)

33. Lin, P., Jia, Y., Du, J., Yu, F.: Distributed leadless coordination for networks of second-order agents with time-delay on switching topology. In: 2008 American Control Conference. pp. 1564-1569. IEEE (2008)

34. Graham, A.: Kronecker products and matrix calculus with applications. Holsted Press, New York (1981) 